ESAIM: PROCEEDINGS, November 2005, Vol.15, 18-57

T. Goudon, E. Sonnendrucker \& D. Talay, Editors

DOI: $10.1051 /$ proc:2005019

\title{
SOME STOCHASTIC PARTICLE METHODS FOR NONLINEAR PARABOLIC PDES
}

\author{
Mireille Bossy ${ }^{1}$
}

\begin{abstract}
We introduce, on some examples, the main tools to analyze the convergence rate of stochastic particle methods for the numerical approximation of some nonlinear parabolic PDEs. We treat the case of Lipschitz coefficients as well as the case of viscous scalar conservation laws. We also discuss some particular aspects like the case of a bounded spatial domain or the use of a Romberg extrapolation as a speed up procedure.
\end{abstract}

Résumé. On introduit, sur certains exemples, les outils principaux pour l'analyse du taux de convergence des méthodes particulaires stochastiques pour l'approximation numérique de quelques EDPs paraboliques non-linéaires. On traite le cas de coefficients lipschitziens, ainsi que le cas des lois de conservation scalaires. On discute quelques aspects particuliers comme le cas d'un domaine spatial borné ou l'utilisation de l'extrapolation de Romberg comme procédé d'accélération.

\section{CONTENTS}

1. Particle approximation for linear parabolic PDEs 19

1.1. The associated martingale problem 19

1.2. Numerical algorithm 21

2. Particle method for the McKean-Valsov model 24

2.1. On the associated particle system 25

2.2. Numerical algorithm 27

2.3. The case of initial bounded signed measure $\quad 30$

3. Some examples 31

3.1. The 2D-incompressible Navier-Stokes equation 31

3.2. The 2D-incompressible Navier-Stokes equation in a bounded domain 33

3.3. Prandtl equation and the vortex sheet method 35

3.4. Interacting particles system in Lagrangian modeling of turbulent flows 37

4. Viscous scalar conservation laws in $\mathbb{R} \quad 39$

4.1. The gradient approach 40

4.2. Numerical comparison of performance 42

4.3. Smoothness of the error with respect to the time step and Romberg extrapolation 45

5. Viscous scalar conservation law in a bounded interval 46

1 INRIA

2004 Route des Lucioles, B.P. 93

06902 Sophia-Antipolis Cedex

France

Mireille.Bossy@sophia.inria.fr 
5.1. Weak solution

\section{Particle approximation for linear parabolic PDEs}

In order to introduce the main ideas of the probabilistic interpretation of nonlinear Fokker-Planck PDEs, let us start with considering a simple linear case. We consider the following PDE, in dimension one, written in the conservative form

$$
\left\{\begin{aligned}
\frac{\partial u_{t}}{\partial t} & =\frac{1}{2} \sigma^{2} \frac{\partial^{2} u_{t}}{\partial x^{2}}-\frac{\partial}{\partial x}\left(B(t, x) u_{t}\right), t>0, x \in \mathbb{R} \\
u_{t=0} & =u_{0} \text { is a given probability measure. }
\end{aligned}\right.
$$

For the time being, we suppose that the convection coefficient $B(t, x)$ is bounded with uniformly bounded first spatial derivative. Equation (1) is a Fokker-Planck equation. From the probabilistic point of view, this means that when the initial condition $u_{0}$ is a probability measure, Equation (1) describes the evolution of the time marginals of the law of a stochastic process.

If $\mu(d x)$ is a bounded measure, for any measurable and bounded function $f$, we use the notation $\langle\mu, f\rangle$ for $\int_{\mathbb{R}} f(x) \mu(d x)$. A weak formulation of Equation (1) is written:

$$
\text { for all } f \in C_{b}^{2}(\mathbb{R}),\left\langle u_{t}, f\right\rangle=\left\langle u_{0}, f\right\rangle+\int_{0}^{t}\left\langle u_{s}, \frac{1}{2} \sigma^{2} f^{\prime \prime}+B(s, \cdot) f^{\prime}\right\rangle d s \text {. }
$$

where $C_{b}^{2}(\mathbb{R})$ is the space of the bounded functions of class $C^{2}$ with bounded derivatives up to the order two.

\subsection{The associated martingale problem}

We want to connect the weak solution of (2) to the law of the stochastic process, solution of

$$
X_{t}=X_{0}+\int_{0}^{t} B\left(s, X_{s}\right) d s+\sigma W_{t}, t \geq 0
$$

where $X_{0}$ is distributed according to the probability measure $u_{0}$ and $\left(W_{t}, t \geq 0\right)$ denotes a one dimensional Brownian motion independent of the initial position $X_{0}$.

One can formulate the existence in law of a diffusion process solution of a stochastic differential equation like $(3)$, in terms of a martingale problem. We introduce the measurable space $(C([0,+\infty) ; \mathbb{R}), \mathcal{B}(C([0,+\infty) ; \mathbb{R})))$ and now we denote $X$ the canonical process on $C([0,+\infty) ; \mathbb{R})$. Finding a solution in law to Equation $(3)$ consists in finding a probability measure $P$ on $(C([0,+\infty) ; \mathbb{R}), \mathcal{B}(C([0,+\infty) ; \mathbb{R})))$ such that the canonical process $\left(X_{t}, t \geq 0\right)$ is a solution of $(3)$. In this formulation, the Brownian motion and the probability space $(\tilde{\Omega}, \tilde{\mathcal{F}}, \tilde{P})$ on which it lives, must be specified in terms of $(C([0,+\infty) ; \mathbb{R}), \mathcal{B}(C([0,+\infty) ; \mathbb{R})), P)$ (see e.g. [22] for more details).

We state the martingale problem related to $(3)$ or $(2)$ :

Definition 1.1. A probability measure $P$ on $(C([0,+\infty) ; \mathbb{R}), \mathcal{B}(C([0,+\infty) ; \mathbb{R})))$ under which

(i) $P \circ X_{0}^{-1}=u_{0}$,

(ii) $\forall f \in C_{b}^{2}(\mathbb{R}), f\left(X_{t}\right)-f\left(X_{0}\right)-\int_{0}^{t}\left[\frac{1}{2} \sigma^{2} f^{\prime \prime}\left(X_{s}\right)+B\left(s, X_{s}\right) f^{\prime}\left(X_{s}\right)\right] d s$ is a $P$-martingale,

is called a solution to the martingale problem $(M P)$ related to (3) or (2) 
The process $\left(M_{t}, t \geq 0\right)$, defined by $M_{t}=f\left(X_{t}\right)-f\left(X_{0}\right)-\int_{0}^{t}\left[\frac{1}{2} \sigma^{2} f^{\prime \prime}\left(X_{s}\right)+B\left(s, X_{s}\right) f^{\prime}\left(X_{s}\right)\right] d s$, is a $P$-martingale if

$$
\mathbb{E}_{P}\left[M_{t}-M_{s} / X_{\theta}, 0 \leq \theta \leq s\right]=0, \forall 0 \leq s \leq t
$$

or equivalently, if for all $g \in C_{b}\left(\mathbb{R}^{n}\right)$ and for all $0 \leq t_{1}<\ldots<t_{n}<s$,

$$
\mathbb{E}_{P}\left[\left(M_{t}-M_{s}\right) g\left(X_{t_{1}}, \ldots X_{t_{n}}\right)\right]=0 .
$$

From a solution of the martingale problem $(M P)$, we can go back to a solution to the Equation (3) in the following manner: applying (ii) for $f(x)=x$, we call $\left(\sigma W_{t}, t \geq 0\right)$ the resulting martingale, with

$$
W_{t}:=\frac{1}{\sigma}\left(X_{t}-X_{0}-\int_{0}^{t} B\left(s, X_{s}\right) d s\right)
$$

Applying (ii) again for $f(x)=x^{2}$, we call $\left(K_{t}\right)_{t \geq 0}$ the resulting martingale. An easy computation shows that

$$
\sigma^{2}\left(W_{t}^{2}-\frac{1}{2} t\right)=K_{t}-2 \sigma X_{0} W_{t}-2 \int_{0}^{t}\left(\int_{0}^{s} B\left(\theta, X_{\theta}\right) d \theta\right) d W_{s}
$$

As the stochastic integral $\left(\int_{0}^{t}\left(\int_{0}^{s} B\left(\theta, X_{\theta}\right) d \theta\right) d W_{s}, t \geq 0\right)$ is a martingale, this last equality identifies $W$ as a Brownian motion, according to the Lévy martingale characterization of Brownian motion. Moreover, taking the expectation in (ii), we get

$$
\mathbb{E} f\left(X_{t}\right)=\mathbb{E} f\left(X_{0}\right)+\int_{0}^{t} \mathbb{E}\left[\frac{1}{2} \sigma^{2} f^{\prime \prime}\left(X_{s}\right)+B\left(s, X_{s}\right) f^{\prime}\left(X_{s}\right)\right] d s .
$$

If $P$ solves $(M P)$, it is a probability measure on $(C([0,+\infty) ; \mathbb{R}), \mathcal{B}(C([0,+\infty) ; \mathbb{R})))$. We denote by $P_{t}$ the marginal at the time $t$ of $P$, that is, the probability measure on $\mathbb{R}$, defined by $P_{t}:=P \circ X_{t}^{-1}$. On the probability space $(C([0,+\infty) ; \mathbb{R}), \mathcal{B}(C([0,+\infty) ; \mathbb{R})), P), P_{t}$ is the law of the random variable $X_{t}$. Then

$$
\mathbb{E} f\left(X_{t}\right)=\int_{\mathbb{R}} f(x) P_{t}(d x)=\left\langle P_{t}, f\right\rangle
$$

and (4) becomes (2):

$$
\left\langle P_{t}, f\right\rangle=\left\langle u_{0}, f\right\rangle+\int_{0}^{t}\left\langle P_{s}, \frac{1}{2} \sigma^{2} f^{\prime \prime}+B(s, \cdot) f^{\prime}\right\rangle d s .
$$

In conclusion, if $P$ solves $(M P)$, then $t \rightarrow P_{t}$ is a weak solution of (1). Conversely, if $u_{t}$ is a weak solution of (1), at each time $t \geq 0$, the measure $u_{t}(d x)$ is the law of $X_{t}$, solution of

$$
X_{t}=X_{0}+\int_{0}^{t} B\left(s, X_{s}\right) d s+\sigma W_{t}, t \geq 0 .
$$

The Lipschitz property of the drift $B(t, x)$ guarantees the existence and uniqueness of the solution $P$ of $(M P)$ (see e.g. [22]). 


\subsection{Numerical algorithm}

To compute $u_{t}$, we follow the probabilistic interpretation. In order to approximate the law of $X_{t}$, it is natural to construct the empirical measure of a sample of independent trials of the random variable $X_{t}$. Let $N$ be the size of the sample. Let $\left(X_{0}^{i}, i=1, \ldots, N\right)$ be $N$ independent random variables of same law $u_{0}$ and $\left(W^{i}, i=1, \ldots, N\right)$ be $N$ independent Brownian motions, independent of $\left(X_{0}^{i}, i=1, \ldots, N\right)$. Let's consider the set of $N$ particles of independent dynamics, given by

$$
X_{t}^{i}=X_{0}^{i}+\int_{0}^{t} B\left(s, X_{s}^{i}\right) d s+\sigma W_{t}^{i}, t \geq 0, i=1, \ldots, N .
$$

We denote by $\bar{U}_{t}^{N}$ the associated empirical measure

$$
\bar{U}_{t}^{N}=\frac{1}{N} \sum_{i=1}^{N} \delta_{X_{t}^{i}} .
$$

If we are interested in a weak approximation of $u_{t}$ then, for any $t \geq 0$, for any $f \in C_{b}(\mathbb{R})$,

$$
\left\langle u_{t}, f\right\rangle-\left\langle\bar{U}_{t}^{N}, f\right\rangle=\mathbb{E} f\left(X_{t}\right)-\frac{1}{N} \sum_{i=1}^{N} f\left(X_{t}^{i}\right) .
$$

The convergence and the associated fluctuation are fully described by the Law of Large Numbers and the Central Limit Theorem. In particular,

$$
\begin{aligned}
\mathbb{E}\left|\mathbb{E} f\left(X_{t}\right)-\frac{1}{N} \sum_{i=1}^{N} f\left(X_{t}^{i}\right)\right| & \leq \sqrt{\mathbb{E}\left(\frac{1}{N} \sum_{i=1}^{N}\left[\mathbb{E} f\left(X_{t}\right)-f\left(X_{t}^{i}\right)\right]\right)^{2}} \\
& \leq \frac{1}{\sqrt{N}} \sqrt{\mathbb{E}\left(f\left(X_{t}\right)-\mathbb{E} f\left(X_{t}\right)\right)^{2}} \leq \frac{1}{\sqrt{N}}\|f\|_{\mathrm{L}^{\infty}(\mathbb{R})} .
\end{aligned}
$$

In our case, given a final date $T>0$, the Fokker-Planck equation (1) has a smooth bounded solution in $(0, T] \times \mathbb{R}$ and we want to construct an approximation function. We denote still by $u_{t}(x)$ the density function of the measure $u_{t}$ :

$$
\forall t>0, u_{t}(d x)=u_{t}(x) d x
$$

We obtain an approximation function by taking the convolution product of the measure $\bar{U}_{t}^{N}$ with a smooth approximation of the Dirac mass, typically a continuous function $\phi$ such that $\int_{\mathbb{R}} \phi(y) d y=1$ and $\phi_{\varepsilon}(x):=\frac{1}{\varepsilon} \phi\left(\frac{x}{\varepsilon}\right)$ :

$$
\bar{U}_{t}^{N, \varepsilon}(x)=\left(\phi_{\varepsilon} * \bar{U}_{t}^{N}\right)(x)=\frac{1}{N} \sum_{i=1}^{N} \phi_{\varepsilon}\left(x-X_{t}^{i}\right) .
$$

The rate of convergence of this smoothing procedure depends on the order of the class of the cutoff function $\phi$. We have the following

Lemma 1.2. [40, Raviart 85] A continuous function $\phi: \mathbb{R} \rightarrow \mathbb{R}$, such that

a) $\int_{\mathbb{R}} \phi(x) d x=1$,

b) $\int_{\mathbb{R}} x^{q} \phi(x) d x=0, \forall q \leq r-1$.

c) $\int_{\mathbb{R}}|x|^{r}|\phi(x)| d x<\infty$. 
is called a cutoff function of order $r$. Then, for any function $v \in W^{r, p}(\mathbb{R}), 1 \leq p \leq \infty$,

$$
\left\|v-\left(v * \phi_{\varepsilon}\right)\right\|_{0, p} \leq C \varepsilon^{r}|v|_{r, p} .
$$

\|\|$_{l, p}$ and ||$_{l, p}$ are the classical notations for the norm and semi-norm associated to the Sobolev space $W^{l, p}(\mathbb{R})$. The proof of Lemma 1.2 is based on the Taylor formula: if $\phi$ is a cutoff function of order $\mathrm{r}$ and $v \in C_{b}^{r}(\mathbb{R})$,

$$
\begin{aligned}
& \left\|v(x)-\left(v * \phi_{\varepsilon}\right)(x)\right\|_{L^{\infty}(\mathbb{R})} \\
& \leq\left\|\frac{1}{(r-1) !} \int_{0}^{1}(1-u)^{r-1}\left(\int_{\mathbb{R}} \frac{\partial^{r} v}{\partial x^{r}}(x+u(y-x))(y-x)^{r} \phi_{\varepsilon}(x-y) d y\right) d u\right\|_{L^{\infty}(\mathbb{R})} \\
& \leq C \varepsilon^{r}\left\|\frac{\partial^{r} v}{\partial x^{r}}\right\|_{L^{\infty}(\mathbb{R})} .
\end{aligned}
$$

Simple examples of cutoff functions are those constructed from Gauss functions. Indeed, in dimension one:

- $\frac{1}{\sqrt{2 \pi}} \exp \left(-\frac{x^{2}}{2}\right)$ is a cutoff function of order 2 .

- $\frac{1}{\sqrt{2 \pi}} \frac{\left(3-x^{2}\right)}{2} \exp \left(-\frac{x^{2}}{2}\right)$ is a cutoff function of order 4 .

Let $\phi(x)$ be a cutoff function of order $r$, which we will choose bounded, in addition. We set

$$
u_{t}^{\varepsilon}(x):=\left(u_{t} * \phi_{\varepsilon}\right)(x)
$$

so that $u_{t}^{\varepsilon}(x)=\mathbb{E} \phi_{\varepsilon}\left(x-X_{t}\right)$. By Lemma 1.2, at a fixed time $t$, we can decompose the error in

$$
\sup _{x \in \mathbb{R}} \mathbb{E}\left|u_{t}(x)-\bar{U}_{t}^{N, \varepsilon}(x)\right| \leq C \varepsilon^{r}+\sup _{x \in \mathbb{R}} \mathbb{E}\left|u_{t}^{\varepsilon}(x)-\bar{U}_{t}^{N, \varepsilon}(x)\right| .
$$

Again, the Law of Large Numbers gives the convergence. More precisely, as the $\left(X_{t}^{i}, i=1, \ldots, N\right)$ are independent,

$$
\begin{aligned}
\mathbb{E}\left|u_{t}^{\varepsilon}(x)-\bar{U}_{t}^{N, \varepsilon}(x)\right| & =\mathbb{E}\left|\frac{1}{N} \sum_{i=1}^{N}\left[\phi_{\varepsilon}\left(x-X_{t}^{i}\right)-\mathbb{E} \phi_{\varepsilon}\left(x-X_{t}\right)\right]\right| \\
& \leq \sqrt{\mathbb{E}\left(\frac{1}{N} \sum_{i=1}^{N}\left[\phi_{\varepsilon}\left(x-X_{t}^{i}\right)-\mathbb{E} \phi_{\varepsilon}\left(x-X_{t}\right)\right]\right)^{2}} \\
& \leq \frac{1}{\sqrt{N}} \sqrt{\mathbb{E}\left(\phi_{\varepsilon}\left(x-X_{t}\right)\right)^{2}} \\
& \leq \frac{1}{\sqrt{N}} \frac{1}{\sqrt{\varepsilon}}\|\phi\|_{\mathrm{L}^{\infty}(\mathbb{R})}^{\frac{1}{2}} \sqrt{\mathbb{E} \phi_{\varepsilon}\left(x-X_{t}\right)}=\frac{1}{\sqrt{N}} \frac{1}{\sqrt{\varepsilon}}\|\phi\|_{\mathrm{L}^{\infty}(\mathbb{R})}^{\frac{1}{2}} \sqrt{\mathbb{E} u_{t}\left(Y^{\varepsilon}-x\right)} \\
& \leq \frac{1}{\sqrt{\varepsilon N}}\|\phi\|_{\mathrm{L}^{\infty}(\mathbb{R})}^{\frac{1}{2}}\left\|u_{t}\right\|_{\mathrm{L}^{\infty}(\mathbb{R})}^{\frac{1}{2}}
\end{aligned}
$$

where $Y^{\varepsilon}$ denotes a random variable of law $\phi_{\varepsilon}(x) d x$. Finally

$$
\sup _{x \in \mathbb{R}} \mathbb{E}\left|u(t, x)-\bar{U}_{t}^{N, \varepsilon}(x)\right| \leq C_{1} \varepsilon^{r}+\frac{C_{2}}{\sqrt{\varepsilon N}} .
$$




\subsubsection{Time discretization of processes $\left(X_{t}^{i}\right)$}

The procedure described above requires trials of the $N$ particles of dynamic (5). The law of the processes (5) is generally unknown. Another approximation is necessary which involves a time discretization scheme. The simplest method for approximating the solution of

$$
X_{t}^{i}=X_{0}^{i}+\int_{0}^{t} B\left(s, X_{s}^{i}\right) d s+\sigma W_{t}^{i}, t \in[0, T], i=1, \ldots, N,
$$

is the Euler scheme. Let $\Delta t$ and $K$ such that $K \Delta t=T ; t_{k}:=k \Delta t$. For any $i=1 \ldots, N$, let's define the discrete time process $\left(\bar{X}_{k \Delta t}^{i}, k=1, \ldots, K\right)$ by

$$
\left\{\begin{array}{l}
\bar{X}_{(k+1) \Delta t}^{i}=\bar{X}_{k \Delta t}^{i}+\Delta t B\left(k \Delta t, \bar{X}_{k \Delta t}^{i}\right)+\sigma\left(W_{(k+1) \Delta t}^{i}-W_{k \Delta t}^{i}\right) \\
\bar{X}_{0}^{i}=X_{0}^{i} \text { of law } u_{0}, i=1, \ldots, N
\end{array}\right.
$$

To simulate a set of the $N$ trajectories of $\left(\bar{X}^{i}, i=1, \ldots, N\right)$, one simply has to simulate the family

$$
\left(W_{\Delta t}^{i}, W_{2 \Delta t}^{i}-W_{\Delta t}^{i}, \ldots, W_{T}^{i}-W_{(K-1) \Delta t}^{i}\right), i=1, \ldots, N
$$

of independent Gaussian random variables. The convergence rate of the Euler scheme has been studied mainly for $\mathrm{L}^{p}(\Omega)$-convergence (called strong convergence) and for convergence of expectation of functionals (weak convergence). For more details, see Talay [43] and the references cited within.

Finally, the approximation function given by a computer is

$$
\bar{U}_{k \Delta t}^{N, \varepsilon, \Delta t}(x)=\frac{1}{N} \sum_{i=1}^{N} \phi_{\varepsilon}\left(x-\bar{X}_{t}^{i}\right)
$$

and the error generated by applying the Euler scheme is bounded as

$$
\begin{aligned}
\mathbb{E}\left|\bar{U}_{k \Delta t}^{N, \varepsilon}(x)-\bar{U}_{k \Delta t}^{N, \varepsilon, \Delta t}(x)\right| \leq & \frac{1}{N} \sum_{i=1}^{N} \mathbb{E}\left|\phi_{\varepsilon}\left(x-X_{k \Delta t}^{i}\right)-\phi_{\varepsilon}\left(x-\bar{X}_{k \Delta t}^{i}\right)\right| \\
& =\mathbb{E}\left|\phi_{\varepsilon}\left(x-X_{k \Delta t}^{1}\right)-\phi_{\varepsilon}\left(x-\bar{X}_{k \Delta t}^{1}\right)\right|
\end{aligned}
$$

by the symmetry of the laws of the $\left(X^{i}\right)$ and $\left(\bar{X}^{i}\right)$. Then,

$$
\sup _{x \in \mathbb{R}} \mathbb{E}\left|\bar{U}_{k \Delta t}^{N, \varepsilon}(x)-\bar{U}_{k \Delta t}^{N, \varepsilon, \Delta t}(x)\right| \leq \sup _{x \in \mathbb{R}}\left|\phi_{\varepsilon}^{\prime}(x)\right| \mathbb{E}\left|X_{k \Delta t}^{1}-\bar{X}_{k \Delta t}^{1}\right| \leq \frac{C}{\varepsilon^{2}} \mathbb{E}\left|X_{k \Delta t}^{1}-\bar{X}_{k \Delta t}^{1}\right|
$$

and

$$
\mathbb{E}\left\|\bar{U}_{k \Delta t}^{N, \varepsilon}(x)-\bar{U}_{k \Delta t}^{N, \varepsilon, \Delta t}(x)\right\|_{L^{1}(\mathbb{R})} \leq\left\|\phi_{\varepsilon}^{\prime}(x)\right\|_{L^{1}(\mathbb{R})} \mathbb{E}\left|X_{k \Delta t}^{1}-\bar{X}_{k \Delta t}^{1}\right| \leq \frac{C}{\varepsilon} \mathbb{E}\left|X_{k \Delta t}^{1}-\bar{X}_{k \Delta t}^{1}\right|
$$

The $\mathrm{L}^{1}(\Omega)$-convergence rate of the Euler scheme $\mathbb{E}\left|X_{k \Delta t}^{1}-\bar{X}_{k \Delta t}^{1}\right|$ depends on the regularity of the drift function $B(t, x)$ in time and space variables. If $B$ is bounded and Lipschitz both in $t$ and $x$, then classical arguments on the analysis of the Euler scheme (see e.g. [43]) lead to

$$
\sup _{k \in\{0, \ldots, K\}} \mathbb{E}\left|X_{k \Delta t}^{1}-\bar{X}_{k \Delta t}^{1}\right| \leq C \Delta t
$$


Thus, for any $t \in[0, T]$, we finally control the global error of the complete approximation procedure with

$$
\begin{aligned}
& \sup _{x \in \mathbb{R}} \mathbb{E}\left|u(t, x)-\bar{U}_{t}^{N, \varepsilon, \Delta t}(x)\right|+\mathbb{E}\left\|u(t, x)-\bar{U}_{t}^{N, \varepsilon, \Delta t}(x)\right\|_{L^{1}(\mathbb{R})} \\
& \leq C\left(\varepsilon^{r}+\frac{1}{\sqrt{\varepsilon N}}+\frac{\Delta t}{\varepsilon^{2}}\right) .
\end{aligned}
$$

The constant $C$ depends on $T$.

\section{Particle method For the McKean-Valsov model}

The McKean-Valsov model is the prototypic model of a particle system in mean field and weak interaction. This means that the system acts over one fixed particle through a smooth function of the empirical measure of the system.

Let us first detail the associated (limit) nonlinear equation. Consider two Lipschitz kernels $b(x, y)$ from $\mathbb{R}^{d} \times \mathbb{R}^{d}$ to $\mathbb{R}^{d}$ and $\sigma(x, y)$ from $\mathbb{R}^{d} \times \mathbb{R}^{d}$ to $\mathcal{L}\left(\mathbb{R}^{k} ; \mathbb{R}^{d}\right)$, the set of matrices $(d \times k)$. For any probability measure $\mu$ on $\mathbb{R}^{d}$, consider the differential operator $\mathcal{L}(\mu)$ defined by

$$
\mathcal{L}(\mu) f(x)=\frac{1}{2} \sum_{i, j=1}^{d} a_{i j}[x, \mu] \frac{\partial^{2} f}{\partial x_{i} \partial x_{j}}(x)+\sum_{i=1}^{d} b_{i}[x, \mu] \frac{\partial f}{\partial x_{i}}(x),
$$

where

$$
\begin{aligned}
& b[x, \mu]:=\int_{\mathbb{R}^{d}} b(x, y) \mu(d y), \\
& a[x, \mu]:=\sigma[x, \mu]^{t} \sigma[x, \mu], \text { with } \sigma[x, \mu]=\int_{\mathbb{R}^{d}} \sigma(x, y) \mu(d y) .
\end{aligned}
$$

The nonlinear partial differential equation, called McKean-Valsov equation, is

$$
\left\{\begin{array}{l}
\frac{\partial U_{t}}{\partial t}=\frac{1}{2} \sum_{i, j=1}^{d} \frac{\partial^{2}}{\partial x_{i} \partial x_{j}}\left(a_{i j}\left[x, U_{t}\right] U_{t}\right)-\sum_{i=1}^{d} \frac{\partial}{\partial x_{i}}\left(b_{i}\left[x, U_{t}\right] U_{t}\right), \text { in }(0,+\infty) \times \mathbb{R}^{d} \\
U_{0} \text { a given probability measure on } \mathbb{R}^{d}
\end{array}\right.
$$

Solution of (10) has been studied from a probabilistic point of view by several authors. One can refer for example to the works of McKean 67 [29], Tanaka 82 [45], Léonard 86 [25], Gärtner 88 [17], Sznitman 89 [42], Méléard 95 [30]. Equation (10) must be considered in a weak sense: we look for $t \rightarrow U_{t}$, probability measure on $\mathbb{R}^{d}$, such that

$$
\forall f \in C_{K}^{\infty}\left(\mathbb{R}^{d}\right), \frac{\partial}{\partial t}\left\langle U_{t}, f\right\rangle=\left\langle U_{t}, \mathcal{L}\left(U_{t}\right) f\right\rangle
$$

Here $C_{K}^{\infty}\left(\mathbb{R}^{d}\right)$ is the space of $C^{\infty}$ functions with compact support. The probabilistic point of view consists in introducing a stochastic process whose time marginals of the distribution are solutions of (11). This process is defined as the solution of a martingale problem.

Definition 2.1. Let $\left(X_{t}, t \in[0, T]\right)$ be the canonical process on $C\left([0, T] ; \mathbb{R}^{d}\right)$. The probability measure $P$ on $C\left([0, T] ; \mathbb{R}^{d}\right)$ is a solution of the martingale problem $(M P)$ issued from a given $U_{0}$ if

$$
\forall f \in C_{b}^{2}\left(\mathbb{R}^{d}\right), f\left(X_{t}\right)-f\left(X_{0}\right)-\int_{0}^{t} \mathcal{L}\left(P_{s}\right) f\left(X_{s}\right) d s \text { is a } P \text {-martingale, }
$$


where $P_{s}=P \circ X_{s}^{-1}$ and where we impose $P_{0}=P \circ X_{0}^{-1}=U_{0}$.

Theorem 2.2. [30, Méléard 95] If $b(\cdot, \cdot)$ and $\sigma(\cdot, \cdot)$ are Lipschitz continuous kernels on $\mathbb{R}^{2 d}$ and if $\mathbb{E}\left|X_{0}\right|^{2}<+\infty$, there exists a unique solution to the martingale problem $(M P)$.

Moreover, there exists a unique solution pathwise (given $X_{0} \in \mathrm{L}^{2}(\Omega)$ and $W$ ) and in law, to the stochastic differential equation,

$$
\begin{aligned}
& X_{t}=X_{0}+\int_{0}^{t} \sigma\left[X_{s}, U_{s}\right] d W_{s}+\int_{0}^{t} b\left[X_{s}, U_{s}\right] d s \\
& \forall t \geq 0, U_{t}=\operatorname{Law}\left(X_{t}\right) .
\end{aligned}
$$

This SDE, whose coefficients depend on the law of the solution, is nonlinear in the sense of McKean and is called a McKean equation.

Taking the expectation in (12), it is not difficult to conclude that $t \rightarrow P_{t}$ is a weak solution of the McKeanVlasov equation (10), where $P_{t}$ are the time marginals of the unique solution $P$ of $(M P)$.

The proof, detailed in [42, Sznitman 91] and [30, Méléard 96], uses a fixed-point technique: let $\mathcal{P}_{2}$ be the space of probability measure on $C\left([0, T], \mathbb{R}^{d}\right)$ such that $\mathbb{E}_{P}\left(\sup _{t \in[0, T]}\left|X_{t}^{2}\right|\right)<\infty$. $\mathcal{P}_{2}$ is endowed with the Vaserstein metric. Consider the map $\Phi$ which associates to $m \in \mathcal{P}\left(C\left([0, T] ; \mathbb{R}^{d}\right)\right)$ the law of the solution of

$$
X_{t}^{m}=X_{0}+\int_{0}^{t} \sigma\left[X_{s}^{m}, m_{s}\right] d W_{s}+\int_{0}^{t} b\left[X_{s}^{m}, m_{s}\right] d s
$$

Existence and uniqueness of the martingale problem $(M P)$ is then translated into a fixed point problem for $\Phi$.

\subsection{On the associated particle system}

If we follow the guideline of the linear case, it seems natural to replace the probability measure $U_{t}=\operatorname{Law}\left(X_{t}\right)$ by the empirical measure $\mu^{N}:=\frac{1}{N} \sum_{i=1}^{N} \delta_{X^{i, N}}$ of particles whose dynamics have to mimic the equation of $\left(X_{t}, t \geq 0\right)$. This leads to the following system of interacting particles

$$
X_{t}^{i, N}=X_{0}^{i}+\int_{0}^{t} \sigma\left[X_{s}^{i, N}, \mu_{s}^{N}\right] d W_{s}^{i}+\int_{0}^{t} b\left[X_{s}^{i, N}, \mu_{s}^{N}\right] d s, t \in[0, T], i=1, \ldots, N .
$$

$\left(W^{1}, \ldots, W^{N}\right)$ is a $d \times N$-dimensional Brownian motion, independent of the initial variables $\left(X_{0}^{1, N}, \ldots, X_{0}^{N, N}\right)$ which are i.i.d. (independent and identically distributed) with law $U_{0}$. The convergence of $\mu_{t}^{N}$ to $U_{t}$ and more generally the convergence of $\mu^{N}=\left(\mu_{t}^{N}, t \in[0, T]\right)$ to $P$, the solution of the martingale problem (12), explained by the Law of Large Number in the linear case, is now explained by the propagation of chaos property of the particle system. Let us introduce this notion with the formal definition given by Sznitman [42].

Definition 2.3. Let $E$ be a separable metric space and $\nu$ a probability measure on $E$. A sequence of symmetric probabilities $\nu^{N}$ on $E^{N}$ is $\nu$-chaotic if for any $\phi_{1}, \ldots, \phi_{k} \in C_{b}(E ; \mathbb{R}), k \geq 1$,

$$
\lim _{N \rightarrow \infty}\left\langle\nu^{N}, \phi_{1} \otimes \ldots \otimes \phi_{k} \otimes 1 \ldots \otimes 1\right\rangle=\prod_{l=1}^{k}\left\langle\nu, \phi_{l}\right\rangle .
$$

Proposition 2.4. [42, Sznitman 89] Let $\mathcal{P}(E)$ denotes the set of probability measure on $E$. Let $\left(X^{i, N}, i=\right.$ $1, \ldots, N)$ be the canonical coordinates on $\left(E^{N}, \nu^{N}\right)$. The empirical measure $\mu^{N}=\frac{1}{N} \sum_{i=1}^{N} \delta_{X^{i, N}}$ is a $\mathcal{P}(E)$ valued random variable on $\left(E^{N}, \nu^{N}\right)$.

" $\nu^{N}$ is $\nu$-chaotic" is equivalent to "the random measure $\mu^{N}$ converges in law to the deterministic value $\nu$ ". 
The propagation of chaos property explains the convergence of the algorithm: when $N$ goes to infinity, any finite subsystem of these particles tends to behave like a system of independent particles, each one having the law $P$, solution of the martingale problem (12).

Theorem 2.5. Let $P^{N}$ be the joint law on $(C([0, T] ; \mathbb{R}))^{N}$ of the particle system $\left(X^{1, N}, \ldots, X^{N, N}\right)$, solution of (14). The sequence $\left(P^{N}\right)$ is $P$-chaotic, where $P$ is the solution of the nonlinear martingale problem (12).

Sketch of the proof. We summarize arguments appearing in [32, Méléard Roelly 87] or [42, Sznitman 91]. According to Proposition 2.4, the P-chaoticity is equivalent to the convergence of the laws of the empirical measures $\mu^{N}$ to $\delta_{P}$.

When the kernels $b(\cdot, \cdot)$ and $\sigma(\cdot, \cdot)$ are smooth, the arguments are as follows. First, one shows that the sequence of the laws of the $\mu^{N}$ 's is tight. Let $\Pi^{\infty}$ be a limit point of a convergent subsequence of $\left\{\operatorname{Law}\left(\mu^{N}\right), N \in \mathbb{N}\right\}$. Set

$$
F(m):=\left\langle m,\left(f\left(X_{t}\right)-f\left(X_{s}\right)-\int_{s}^{t} \mathcal{L}\left(m_{\theta}\right) f\left(X_{\theta}\right) d \theta\right) g\left(X_{s_{1}}, \ldots, X_{s_{k}}\right)\right\rangle
$$

where $\mathcal{L}(\mu)$ is as in $(7), f \in C_{b}^{2}(\mathbb{R}), g \in C_{b}\left(\mathbb{R}^{k}\right), 0 \leq s_{1}<\ldots<s_{k}<s \leq T$ and $m$ is a probability on $C([0, T] ; \mathbb{R})$. Then one uses two arguments:

(a) first, one checks that $\lim _{N \rightarrow+\infty} \mathbb{E}\left[F\left(\mu^{N}\right)\right]^{2}=0$ by using the dynamics of the particles.

(b) Then, one uses the continuity of $F(\cdot)$ in $\mathcal{P}(C([0, T] ; \mathbb{R}))$ endowed with the Vaserstein metric to deduce that the support of $\Pi^{\infty}$ is the set of solutions to the nonlinear martingale problem (12). One proves the uniqueness of such a solution, which implies that $\Pi^{\infty}=\delta_{P}$.

In the system (14), the initials positions $\left(X_{0}^{1, N}, \ldots, X_{0}^{N, N}\right)$ are i.i.d. with law $U_{0}$. Then it is clear that the initial laws $P_{0}^{N}=\operatorname{Law}\left(X_{0}^{1, N}, \ldots, X_{0}^{N, N}\right)$ are $U_{0}$-chaotic. Theorem 2.5 states that the initial chaos propagates in time. By Definition 2.3, for any $\phi \in C_{b}\left(\mathbb{R}^{d}\right)$, at any time $t \in[0, T]$,

$$
\lim _{N \rightarrow \infty}\left\langle\mu_{t}^{N}, \phi\right\rangle=\left\langle P_{t}, \phi\right\rangle=\mathbb{E} \phi\left(X_{t}\right) .
$$

The convergence could also be studied by coupling the particle system with a set of independent and linear SDEs, but depending on the time marginals $P_{t}$ of the solution on the martingale problem:

$$
X_{t}^{i}=X_{0}^{i}+\int_{0}^{t} \sigma\left[X_{s}^{i}, P_{s}\right] d W_{s}^{i}+\int_{0}^{t} b\left[X_{s}^{i}, P_{s}\right] d s, \operatorname{Law}\left(X_{0}^{i}\right)=U_{0}, i=1, \ldots, N .
$$

Then

Theorem 2.6. [30, Méléard 95] $\sup _{N} \mathbb{E}\left(\sup _{0 \leq t \leq T}\left|X_{t}^{i, N}\right|^{2}\right)+\sup _{N} \mathbb{E}\left(\sup _{0 \leq t \leq T}\left|X_{t}^{i}\right|^{2}\right)<\infty$ and

$$
\sup _{N} N \mathbb{E}\left(\sup _{0 \leq t \leq T}\left|X_{t}^{i, N}-X_{t}^{i}\right|^{2}\right)<\infty
$$

Sketch of the proof. This proof is also detailed in [42, Sznitman 89] when $\sigma$ is a constant and $b$ is a Lipschitz bounded kernel. Let us consider this particular situation. Then,

$$
X_{t}^{i, N}-X_{t}^{i}=\int_{0}^{t}\left[\frac{1}{N} \sum_{j=1}^{N} b\left(X_{s}^{i, N}, X_{s}^{j, N}\right)-\int_{\mathbb{R}^{d}} b\left(X_{s}^{i}, y\right) P_{s}(d y)\right] d s
$$


and

$$
\sum_{i=1}^{N} \mathbb{E} \sup _{t \in[0, T]}\left|X_{t}^{i, N}-X_{t}^{i}\right| \leq \int_{0}^{T}\left(2 K \sum_{i=1}^{N} \mathbb{E}\left|X_{s}^{i, N}-X_{s}^{i}\right|+\sum_{i=1}^{N} \mathbb{E}\left|\frac{1}{N} \sum_{j=1}^{N} \beta_{s}\left(X_{s}^{i}, X_{s}^{j}\right)\right|\right) d s
$$

where $K$ is the Lipschitz constant of the kernel $b(\cdot, \cdot)$ and where we set $\beta_{s}(x, y):=b(x, y)-\int_{\mathbb{R}^{d}} b(x, z) P_{s}(d z)$. By symmetry, for all $i=1, \ldots, N$,

$$
\mathbb{E} \sup _{t \in[0, T]}\left|X_{t}^{i, N}-X_{t}^{i}\right| \leq \int_{0}^{T} 2 K \mathbb{E}\left|X_{s}^{i, N}-X_{s}^{i}\right|+\mathbb{E}\left|\frac{1}{N} \sum_{j=1}^{N} \beta_{s}\left(X_{s}^{i}, X_{s}^{j}\right)\right| d s, \forall i=1, \ldots, N
$$

Applying the Grownwall lemma, it comes that

$$
\mathbb{E} \sup _{t \in[0, T]}\left|X_{t}^{i, N}-X_{t}^{i}\right| \leq C \int_{0}^{T} \mathbb{E}\left|\frac{1}{N} \sum_{j=1}^{N} \beta_{s}\left(X_{s}^{i}, X_{s}^{j}\right)\right| d s
$$

But,

$$
\frac{1}{N} \sum_{j=1}^{N} \beta_{s}\left(X_{s}^{i}, X_{s}^{j}\right)=\frac{1}{N} \sum_{j=1}^{N}\left[b\left(X_{s}^{i}, X_{s}^{j}\right)-\left.\mathbb{E} b\left(x, X_{s}\right)\right|_{x=X_{s}^{i}}\right]
$$

is a sum of centered random variables. Then, its expectation is bounded by $C / \sqrt{N}$.

\subsection{Numerical algorithm}

In this subsection, the state space is $\mathbb{R}$ (i.e. $d=1$ ).

Let $v(t, x)$ be the cumulative distribution function defined by $\left.\left.v(t, x)=P_{t}(]-\infty, x\right)\right)=\mathbb{E} H\left(X_{t}-x\right), X$ solving (13). Here, $H(x)=\mathbb{1}_{\{x \geq 0\}}$ denotes the Heaviside function. The cumulative distribution function solves

$$
\left\{\begin{array}{l}
\frac{\partial v}{\partial t}(t, x)=\frac{1}{2} \frac{\partial}{\partial x}\left(a\left[x, \frac{\partial v}{\partial x}(t, \cdot)\right] \frac{\partial v}{\partial x}(t, x)\right)-b\left[x, \frac{\partial v}{\partial x}(t, \cdot)\right] \frac{\partial v}{\partial x}(t, x),(t, x) \in(0, T] \times \mathbb{R} \\
\left.\left.v(0, x)=U_{0}(]-\infty, x\right)\right), x \in \mathbb{R}
\end{array}\right.
$$

We construct an approximation method for the solutions of (15) and (10), based upon the time discretization of the system (14). From now on, the number $N$ of particles is fixed.

We suppose that the following assumptions hold:

(H1) There exists a strictly positive constant $s_{*}$ such that $s(x, y) \geq s_{*}>0, \forall(x, y)$.

(H2) The kernels $b(\cdot, \cdot)$ and $s(\cdot, \cdot)$ are uniformly bounded functions on $\mathbb{R}^{2} ; b(\cdot, \cdot)$ is globally Lipschitz and $s(\cdot, \cdot)$ has uniformly bounded first partial derivatives.

(H3) The initial law $U_{0}$ satisfies:

(i) either $U_{0}$ is a Dirac measure at $x_{0}$, or

(ii) $U_{0}$ has a continuous density $u_{0}$ satisfying: there exist constants $M>0, \eta \geq 0$ and $\alpha>0$ such that $u_{0}(x) \leq \eta \exp \left(-\alpha \frac{x^{2}}{2}\right)$ for $|x|>M$. If $\eta=0, U_{0}$ has compact support. 
(H1) and (H2) imply that, for all $t>0$, the solution of the McKean-Vlasov equation (10) has a density $u(t, \cdot)$ with respect to Lebesgue measure. Moreover $(\mathrm{H} 1)$ and (H2) allow to get exponential estimates one $u(t, \cdot)$, useful for the proof of the convergence rate.

The algorithm starts with an approximation of the initial condition $v(0, \cdot)$ of $(15)$. The $N$ points $\left(y_{0}^{1}, \ldots, y_{0}^{N}\right)$ are chosen in $\mathbb{R}$ such that the piecewise constant function

$$
\bar{v}_{0}(x)=\frac{1}{N} \sum_{i=1}^{N} H\left(x-y_{0}^{i}\right)
$$

approximates $v_{0}$ in $\mathrm{L}^{1}(\mathbb{R})$ with a sufficiently high accuracy. If $U_{0}$ is a Dirac measure at $x_{0}$, the $N$ particles are located at $y_{0}^{i}=x_{0}$ and $\bar{v}_{0}(\cdot)=v_{0}(\cdot)$. When $U_{0}$ satisfies (H3-ii), one can choose deterministic initial positions

$$
y_{0}^{i}= \begin{cases}\inf \left\{y ; v_{0}(y)=\frac{i}{N}\right\}, & i=1, \ldots, N-1, \\ \inf \left\{y ; v_{0}(y)=1-\frac{1}{2 N}\right\}, & i=N .\end{cases}
$$

We set $\bar{U}_{0}:=\frac{1}{N} \sum_{i=1}^{N} \delta_{y_{0}^{i}}$. Consider the system (14) with the initial condition $X_{0}^{i, N}=y_{0}^{i}$ and denote its solution by $\left(X_{t}^{i}, 1 \leq i \leq N\right)$. There holds

$$
\left\{\begin{array}{l}
d X_{t}^{i}=\frac{1}{N} \sum_{j=1}^{N} b\left(X_{t}^{i}, X_{t}^{j}\right) d t+\frac{1}{N} \sum_{j=1}^{N} s\left(X_{t}^{i}, X_{t}^{j}\right) d W_{t}^{i}, t \in[0, T] \\
X_{0}^{i}=y_{0}^{i}, i=1, \ldots, N
\end{array}\right.
$$

The propagation of chaos suggests that $1 / N \sum_{i=1}^{N} \delta_{X_{t}^{i}}$ approximates the solution $U_{t}$ of Equation (10). To get a simulation procedure for a trajectory of each $\left(X_{t}^{i}\right)$, we discretize in time: $\Delta t>0$ and $K \in \mathbb{N}$ are chosen such that $T=K \Delta t$; the discrete times are denoted by $t_{k}:=k \Delta t$, with $1 \leq k \leq K$. The Euler scheme leads to the following discrete-time system:

$$
\left\{\begin{array}{l}
Y_{t_{k+1}}^{i}=Y_{t_{k}}^{i}+\frac{1}{N} \sum_{j=1}^{N} b\left(Y_{t_{k}}^{i}, Y_{t_{k}}^{j}\right) \Delta t+\frac{1}{N} \sum_{j=1}^{N} s\left(Y_{t_{k}}^{i}, Y_{t_{k}}^{j}\right)\left(W_{t_{k+1}}^{i}-W i_{t_{k}}\right) \\
Y_{0}^{i}=y_{0}^{i}, i=1, \ldots, N .
\end{array}\right.
$$

Thus, we approximate $U_{t_{k}}$ by the empirical measure

$$
\bar{U}_{t_{k}}:=\frac{1}{N} \sum_{i=1}^{N} \delta_{Y_{t_{k}}^{i}} .
$$

In the same way, we approximate $v\left(t_{k}, \cdot\right)$, solution to (15), by the cumulative distribution function

$$
\bar{v}_{t_{k}}(x):=\frac{1}{N} \sum_{i=1}^{N} H\left(x-Y_{t_{k}}^{i}\right), \forall x \in \mathbb{R}
$$


Theorem 2.7. [9, Bossy and Talay 97] Assume (H1), (H2) and (H3). There exists a strictly positive constant $C$, depending on $s, b, U_{0}$ and $T$, such that for all $k \in\{1, \ldots, K\}$ one has

$$
\begin{aligned}
& \mathbb{E}\left\|v\left(t_{k}, \cdot\right)-\bar{v}_{t_{k}}(\cdot)\right\|_{\mathrm{L}^{1}(\mathbb{R})} \leq C\left(\left\|v_{0}-\bar{v}_{0}\right\|_{\mathrm{L}^{1}(\mathbb{R})}+\frac{1}{\sqrt{N}}+\sqrt{\Delta t}\right) \\
\text { and } \quad & \operatorname{Var}\left(\left\|v\left(t_{k}, \cdot\right)-\bar{v}_{t_{k}}(\cdot)\right\|_{\mathrm{L}^{1}(\mathbb{R})}\right) \leq C\left(\left\|v_{0}-\bar{v}_{0}\right\|_{\mathrm{L}^{1}(\mathbb{R})}^{2}+\frac{1}{N}+\Delta t\right) .
\end{aligned}
$$

Furthermore, $\left\|v_{0}-\bar{v}_{0}\right\|_{\mathrm{L}^{1}(\mathbb{R})} \leq C \sqrt{\log (N)} / N$, where $C$ depends on $M, \eta$ and $\alpha$.

In order to obtain an approximation of the density $u(t, x)$, we construct a smoothing by convolution of the discrete measure $\bar{U}_{t_{k}}$ : let $\phi_{\varepsilon}$ be the density of the Gaussian law $\mathcal{N}\left(0, \varepsilon^{2}\right)$ and set

$$
\bar{u}_{t_{k}}^{\varepsilon}(x):=\left(\phi_{\varepsilon} * \bar{\mu}_{t_{k}}\right)(x)=\frac{1}{N} \sum_{i=1}^{N} \frac{1}{\sqrt{2 \pi} \varepsilon} \exp \left(-\frac{\left(x-Y_{t_{k}}^{i}\right)^{2}}{2 \varepsilon^{2}}\right) .
$$

The hypotheses are strengthen as follows:

(H2') The kernel $b$ is in $C_{b}^{2}\left(\mathbb{R}^{2}\right)$ and $s$ is in $C_{b}^{3}\left(\mathbb{R}^{2}\right)$.

(H3') The initial law $U_{0}$ has a strictly positive density $u_{0}$ in $C^{2}(\mathbb{R})$ satisfying: there exist strictly positive constants $M, \eta$ and $\alpha$ such that

$$
u_{0}(x)+\left|u_{0}^{\prime}(x)\right|+\left|u_{0}^{\prime \prime}(x)\right| \leq \eta \exp \left(-\alpha \frac{x^{2}}{2}\right) \text { for }|x|>M
$$

Under (H1) and (H2'), one can show that the density $u(t, \cdot)$ belongs to the Sobolev space $W^{2,1}(\mathbb{R})$. According to Lemma 1.2, the smoothing error $\left\|u(t, \cdot)-\left(u(t, \cdot) * \phi_{\varepsilon}\right)\right\|_{\mathrm{L}^{1}(\mathbb{R})}$ is of order $\mathcal{O}\left(\varepsilon^{2}\right)$.

Theorem 2.8. [9, Bossy and Talay 97] Assume (H1), (H2') and (H3'). Let $u(t, \cdot)$ be the classical solution to (10). Then there exists a strictly positive constant $C$, depending on $s, b, u_{0}$ and $T$, such that for all $k \in\{1, . ., K\}$ one has

$$
\begin{aligned}
& \mathbb{E}\left\|u\left(t_{k}, \cdot\right)-\bar{u}_{t_{k}}^{\varepsilon}(\cdot)\right\|_{\mathrm{L}^{1}(\mathbb{R})} \leq C\left[\varepsilon^{2}+\frac{1}{\varepsilon}\left(\left\|v_{0}-\bar{v}_{0}\right\|_{\mathrm{L}^{1}(\mathbb{R})}+\frac{1}{\sqrt{N}}+\sqrt{\Delta t}\right)\right] \\
& \text { and } \quad \operatorname{Var}\left(\left\|u\left(t_{k}, \cdot\right)-\bar{u}_{t_{k}}^{\varepsilon}(\cdot)\right\|_{\mathrm{L}^{1}(\mathbb{R})}\right) \leq C\left[\varepsilon^{4}+\frac{1}{\varepsilon^{2}}\left(\left\|v_{0}-\bar{v}_{0}\right\|_{\mathrm{L}^{1}(\mathbb{R})}^{2}+\frac{1}{N}+\Delta t\right)\right] .
\end{aligned}
$$

In the two previous theorems, the theoretical estimates on the rate of convergence in time is of order $\mathcal{O}(1 / 2)$. This is due to the use of the $\mathrm{L}^{2}(\Omega)$-convergence rate of the Euler scheme. Those estimates are too crude and likely hides an additional averaging effect of the large number of particles.

The first work on the optimal rate of convergence of the Euler scheme for interacting particle systems is due to Kohatsu-Higa and Ogawa [23]. They analyze the convergence of the weak approximation of a general nonlinear diffusion process of the form:

$$
\left\{\begin{array}{l}
d X_{t}=a\left(X_{t}, F * u_{t}\left(X_{t}\right)\right) d t+b\left(X_{t}, G * u_{t}\left(X_{t}\right)\right) d W_{t}, \text { where } u_{t} \text { is the law of } X_{t}, \\
X_{t=0}=X_{0} \text { with law } U_{0} .
\end{array}\right.
$$

Assuming that the functions $a, b, F$ and $G$ are smooth with bounded derivatives, the authors use Malliavin calculus to show that, for any $C^{\infty}$ function $f$ whose derivatives have polynomial growth at infinity,

$$
\mathbb{E}\left|\frac{1}{N} \sum_{i=1}^{N} f\left(\bar{X}_{k \Delta t}^{i}\right)-\mathbb{E} f\left(X_{t_{k}}\right)\right| \leq C\left(\frac{1}{\sqrt{N}}+\Delta t\right),
$$


where $C$ is independent of $\Delta t$ and $N$ but depends on $f$ and $\left(\bar{X}_{k \Delta t}^{i}, i=1, \ldots, N\right)$ is the corresponding discrete time system of interacting particles.

More recently Antonelli and Kohatsu-Higa, still by using Malliavin calculus, proved the following optimal rate of convergence result:

Theorem 2.9. [1, Antonelli Kohatsu-Higa 02] If $b(\cdot, \cdot)$ and $\sigma(\cdot, \cdot)$ are $C^{\infty}$ kernels with all bounded derivatives, if the diffusion kernel $\sigma(\cdot, \cdot)$ satisfies the so-called restricted Hörmander condition and if we assume (H3), then

$$
\begin{gathered}
\mathbb{E}\left\|v\left(t_{k}, \cdot\right)-\bar{v}_{t_{k}}(\cdot)\right\|_{\mathrm{L}^{1}(\mathbb{R})} \leq C_{b, \sigma, T, U_{0}}\left(\frac{1}{\sqrt{N}}+\Delta t\right) \\
\text { and } \quad \mathbb{E}\left\|\left(\phi_{\sqrt{\Delta t}} * \bar{U}_{t_{k}}\right)(\cdot)-u\left(t_{k}, \cdot\right)\right\|_{\mathrm{L}^{1}(\mathbb{R})} \leq C_{b, \sigma, T, U_{0}}\left(\frac{1}{\sqrt{N} \Delta t^{\frac{1}{4}}}+\Delta t+\frac{1}{\sqrt{N}}\right) .
\end{gathered}
$$

where $\phi_{\sqrt{\Delta t}}(x)$ is the density of the Gaussian law $\mathcal{N}(0, \Delta t)$.

\subsection{The case of initial bounded signed measure}

Deterministic particle methods for linear hyperbolic equations, like convection problems, do not care about what ever are the sign and the total mass of the initial measure. The initial data is approximated by a linear combination of weighted Dirac measures and particles are described by the couples weight-position $\left(\omega_{i}, X^{i}(t)\right)$, the weights being constant in time.

In parabolic problem, the main difference resides in the fact that particles move according to a system of stochastic differential equation. The weights must still be determined by the measure approximation of the initial condition. To give a probabilistic interpretation of McKean-Valsov equation in that case, the main problem is: how could we integrate signed measures in the martingale problem (12)? An answer is proposed by Jourdain in [19]: let $\mathcal{M}\left(\mathbb{R}^{d}\right)$ be the space of bounded signed measures on $\mathbb{R}^{d}$. For $u \in \mathcal{M}\left(\mathbb{R}^{d}\right)$, let $\mathcal{L}(u)$ denotes the second order differential operator

$$
\mathcal{L}(u) f(x)=\frac{1}{2} \sum_{i, j=1}^{d} a_{i j}[x, u] \frac{\partial^{2} f}{\partial x_{i} \partial x_{j}}+\sum_{i=1}^{d} b_{i}[x, u] \frac{\partial f}{\partial x_{i}}
$$

with $a[x, u]$ and $b[x, u]$ still defined as in (8) but with a measure $u$ in $\mathcal{M}\left(\mathbb{R}^{d}\right) . b(\cdot, \cdot)$ and $\sigma(\cdot, \cdot)$ are bounded and Lipschitz continuous mappings on $\mathbb{R}^{2 d}$ with values in $\mathbb{R}^{d}$ and the space of $d \times k$ real matrices respectively. Consider the McKean-Valsov equation

$$
\left\{\begin{array}{l}
\frac{\partial U_{t}}{\partial t}=\mathcal{L}\left(U_{t}\right) U_{t} \text { in }(0,+\infty) \times \mathbb{R}^{d} \\
U_{0} \neq 0 \text { in } \mathcal{M}\left(\mathbb{R}^{d}\right)
\end{array}\right.
$$

Let $h: \mathbb{R}^{d} \rightarrow\left\{-\left\|U_{0}\right\|,\left\|U_{0}\right\|\right\}$ be a density of the initial measure $U_{0}$ with respect to the probability measure $\frac{\left|U_{0}\right|}{\left\|U_{0}\right\|}$. Here $\left|U_{0}\right|$ is the absolute value of $U_{0}$ and $\left\|U_{0}\right\|$ the total mass of $\left|U_{0}\right|$. For any probability measure $P \in \mathcal{P}\left(C\left([0,+\infty) ; \mathbb{R}^{d}\right)\right)$ we define $\widetilde{\mathcal{P}}_{t}$ in $\mathcal{M}\left(\mathbb{R}^{d}\right)$ by

$$
\widetilde{\mathcal{P}}_{t}(B)=\mathbb{E}_{P}\left(\mathbb{1}_{B}\left(X_{t}\right) h\left(X_{0}\right)\right), \text { for any Borel set } B \in \mathcal{B}\left(\mathbb{R}^{d}\right) .
$$

$X$ is the canonical process on $C\left([0,+\infty) ; \mathbb{R}^{d}\right)$. Now consider the following nonlinear martingale problem: $P \in \mathcal{P}\left(C\left([0,+\infty) ; \mathbb{R}^{d}\right)\right)$ solves the martingale problem $(M P)$ issued from $\frac{\left|U_{0}\right|}{\left\|U_{0}\right\|}$ if

(i) $P \circ X_{0}^{-1}=\left|U_{0}\right| /\left\|U_{0}\right\|$. 
(ii) For any $f \in C_{b}^{2}\left(\mathbb{R}^{d}\right)$,

$$
f\left(X_{t}\right)-f\left(X_{0}\right)-\int_{0}^{t} \mathcal{L}\left(\widetilde{P}_{s}\right) f\left(X_{s}\right) d s \text { is a } P \text {-martingale. }
$$

Then, for any solution $P$ of this problem, $t \rightarrow \widetilde{P}_{t}$ is a weak solution of (17). The McKean equation corresponding to $(17)$ is

$$
\left\{\begin{array}{l}
X_{t}=X_{0}+\int_{0}^{t} \sigma\left[X_{s}, \widetilde{P}_{s}\right] d W_{s}+\int_{0}^{t} b\left[X_{s}, \widetilde{P}_{s}\right] d s \\
\text { where } P \text { is the distribution of } X
\end{array}\right.
$$

Existence and uniqueness of this McKean equation is obtain by adapting the fixed-point technique of Sznitman $[19,42]$.

\section{Some examples}

\subsection{The 2D-incompressible Navier-Stokes equation}

Let us consider the two dimensional Navier-Stokes equation in the whole space:

$$
\begin{aligned}
\partial_{t} \mathcal{U}(t, x)+(\mathcal{U}(t, x) \cdot \nabla) \mathcal{U}(t, x)+\nabla p(t, x) & =\nu \Delta \mathcal{U}(t, x), t \in(0 . T], x=\left(x_{1}, x_{2}\right) \in \mathbb{R}^{2}, \\
\nabla \cdot \mathcal{U}(t, x) & =\partial_{x_{1}} u(t, x)+\partial_{x_{2}} v(t, x)=0, \quad(t, x) \in[0, T] \times \mathbb{R}^{2} \\
\mathcal{U}(0, x) & =\mathcal{U}_{0}(x), x \in \mathbb{R}^{2} .
\end{aligned}
$$

where $\mathcal{U}(t, x)=(u(t, x), v(t, x))$ denotes the velocity of the fluid and $p(t, x)$ is the pressure. It is understood that $\mathcal{U}(\cdot, x) \rightarrow(0,0)$ as $|x| \rightarrow+\infty$. In its vorticity form, Equations (18) have a probabilistic interpretation. The vorticity $\omega$ is defined by

$$
\omega(t, x)=\nabla \times \mathcal{U}(t, x)
$$

which, in two dimensions, is usually considered as the scalar $\omega=\partial_{x_{1}} v-\partial_{x_{2}} u$. Given the vorticity, we want to reconstruct the velocity. As $\nabla \cdot \mathcal{U}=0$, classical results insure the existence of a stream function $\psi$ such that $\mathcal{U}=\nabla^{\perp} \psi=\left(\partial_{x_{2}} \psi,-\partial_{x_{1}} \psi\right)$. Note that $\psi$ can be found by solving the Poisson equation

$$
\begin{aligned}
& \Delta \psi(t, x)=-\omega(t, x), t \in(0, T], x \in \mathbb{R}^{2} \\
& \nabla \psi \rightarrow 0 \text { as }|x| \rightarrow+\infty
\end{aligned}
$$

If $G(x)$ denotes the Green's function for this Poisson equation, then $\psi(t, x)=(G * \omega)(t, x)$ and $\mathcal{U}$ is given by the Biot-Savart law

$$
\mathcal{U}(t, x)=(\mathbf{K} * \omega)(t, x)
$$

where $\mathbf{K}=\nabla \times G$. In two dimension, $\mathbf{K}$ is the vector

$$
\mathbf{K}(x)=\frac{x^{\perp}}{2 \pi|x|^{2}}
$$


By taking the curl of Equations (18a,18c), we obtain the vorticity form of the Navier-Stokes equation

$$
\begin{aligned}
\frac{\partial \omega}{\partial t}(t, x)+\nabla \cdot(\omega(t, x)(\mathbf{K} * \omega)(t, x)) & =\nu \Delta \omega(t, x), t \in(0, T], x \in \mathbb{R}^{2}, \\
\mathcal{U}(t, x) & =(\mathbf{K} * \omega)(t, x), t \in(0, T], x \in \mathbb{R}^{2}, \\
\omega(0, x) & =\omega_{0}(x):=\left(\nabla \times \mathcal{U}_{0}\right)(x), x \in \mathbb{R}^{2} .
\end{aligned}
$$

The vorticity $\omega$ is then solution of a McKean-Vlasov equation like (10) with a constant diffusion $\sigma(x, y):=\sqrt{2 \nu}$ and a singular interacting kernel $b(x, y)=\mathbf{K}(x-y)$. The McKean process $\left(X_{t}, t \geq 0\right)$, associated with (19) solves

$$
\begin{aligned}
& d X_{t}=\sqrt{2 \nu} d W_{t}+\mathcal{U}\left(t, X_{t}\right) d t, t \in(0, T] \\
& \mathcal{U}(t, x)=\left(\mathbf{K} * \omega_{t}\right)(x), \omega_{t}=\operatorname{Law}\left(X_{s}\right)
\end{aligned}
$$

and the corresponding $N$-particle system is describe by

$$
d X_{t}^{i}=\sqrt{2 \nu} d W_{t}^{i}+\frac{1}{N-1} \sum_{j \neq i, j=1}^{N} \mathbf{K}\left(X_{t}^{i}-X^{j}\right) d t, 1 \leq i \leq N
$$

$W$ is a 2 -dimensional Brownian motion and $\left(W^{1}, \ldots, W^{N}\right)$ is a $2 \times N$-dimensional Brownian motion. Due to the singularity of $\mathbf{K}$, specific existence and uniqueness results for (20) and (21) must be established.

In this context, the stochastic particle method corresponds to the well-known random vortex method introduced by Chorin $[12,13]$. The convergence of the random vortex method for the Navier-Stokes equations has been studied firstly from the splitting point of view. Vortex methods denotes a class of numerical methods for approximating the solution of the incompressible Navier Stokes or Euler equations $(\nu=0)$. The splitting method (or fractional step method) consists in writing the algorithm as a numerical procedure, solving successively during the same time step the convection operator in $(19), \frac{\partial \omega}{\partial t}+\nabla \cdot(\omega(\mathbf{K} * \omega))=0$, and the diffusion operator $\frac{\partial \omega}{\partial t}=\nu \Delta \omega$. For the convergence, we refer for example to the works of Beale and Majda [2,3] and Goodman [18]. A rate of convergence result was obtained by Long in [27]. We detail a little this result. Suppose that $\omega(0, x)$ has a compact support. The spatial discretization parameter is not directly the number of particles but the corresponding size $h$ of an initial regular mesh on $\mathbb{R}^{2}$. Indeed, for a fixed $h$, let $\Lambda^{h}=\left\{h . i, i \in \mathbb{Z}^{2}\right\}$. Let $\omega_{0}^{h}(x)$ be the particle approximation of $\omega_{0}$ on the mesh $\Lambda^{h}$, namely

$$
\omega_{0}^{h}(x)=\sum_{\Lambda^{h} \cap \operatorname{Supp}\left(\mathcal{U}_{0}\right)} h^{2} w_{i} \delta_{\left(x-\alpha_{i}\right)}, \text { with } w_{i}=\omega_{0}\left(\alpha_{i}\right) .
$$

Let $\phi \in C^{L}\left(\mathbb{R}^{2}\right)$ a cutoff function of order $m$, decreasing rapidly at infinity. The kernel $\mathbf{K}$ is replaced by a mollified one, $\mathbf{K}_{\varepsilon}=\mathbf{K} * \phi_{\varepsilon}$. Then, the stochastic particle system is

$$
d X_{t}^{\varepsilon, h, i}=\sum_{j} w_{j} h^{2} \mathbf{K}_{\varepsilon}\left(X_{t}^{\varepsilon, h, i}-X_{t}^{\varepsilon, h, j}\right) d t+\sqrt{2 \nu} d W_{t}^{i}
$$

and the corresponding approximation of the velocity field is

$$
\mathcal{U}^{\varepsilon, h}(t, x)=\sum_{j} w_{j} h^{2} \mathbf{K}_{\varepsilon}\left(x-X_{t}^{\varepsilon, h, j}\right)
$$

The following result is extract from Long [27]: 
Theorem 3.1. Let $\varepsilon \geq h^{q}$ with $q<1$. Assume that the velocity field $\mathcal{U}(t, x)$ is smooth enough. Then

$$
\sup _{0 \leq t \leq T}\left\|\mathcal{U}^{\varepsilon, h}(t, \cdot)-\mathcal{U}(t, \cdot)\right\|_{L^{p}\left(B\left(R_{0}\right)\right)} \leq C\left(\varepsilon^{m}+\left(\frac{h}{\varepsilon}\right)^{L} \varepsilon+h|\ln h|\right)
$$

except for an event of probability less than $h^{C^{\prime} C}$, provided that $C>C^{\prime \prime}$ and $C^{\prime}, C^{\prime \prime}>0$ depend only on the data parameters and on the bounds for a finite number of derivatives of $\mathcal{U}(t, x)$.

In the same time, several authors have been interested in the probabilistic interpretation of the vortex equation. Marchioro and Pulvirenti presented first the propagation of chaos problem in [28] and give a partial probabilistic interpretation for the equation with a mollified kernel $\mathbf{K}_{\varepsilon}$. They proved the convergence of the empirical measure of the $N$-particle system, $N \rightarrow+\infty, \varepsilon(N) \rightarrow 0$. Next, Osada, in [35], proved a propagation of chaos result for the interacting particle system (21) only for the case of large viscosity and for a bounded density initial data. More recently, Méléard in [31], generalizes the pathwise proof of the random vortex method (i.e. the convergence of the empirical measures of the particle system, considered as probability measure on the path space, to the solution of the vortex equation) for any viscosity and for a large class of initial data. In [16], Fontbona extends this probabilistic approach for the three dimensional Navier-Stokes equation.

\subsection{The 2D-incompressible Navier-Stokes equation in a bounded domain}

Let $\mathcal{O}$ be a bounded domain in $\mathbb{R}^{2}$. Let us consider now the two dimensional Navier-Stokes equation in $\mathcal{O}$

$$
\begin{aligned}
\partial_{t} \mathcal{U}(t, x)+(\mathcal{U}(t, x) \cdot \nabla) \mathcal{U}(t, x)+\nabla p(t, x) & =\nu \Delta \mathcal{U}(t, x), t>0, x \in \mathcal{O}, \\
\nabla \cdot \mathcal{U}(t, x) & =\partial_{x_{1}} u(t, x)+\partial_{x_{2}} v(t, x)=0, t \geq 0, x \in \mathcal{O}, \\
\mathcal{U}(0, x) & =\mathcal{U}_{0}(x), x \in \partial \mathcal{O} \\
\mathcal{U}(t, x) & =0, t \geq 0, x \in \partial \mathcal{O} .
\end{aligned}
$$

As before, taking the curl of equations $(22 \mathrm{a}, 22 \mathrm{c})$, we obtain the vorticity form of the equation

$$
\begin{aligned}
\frac{\partial \omega}{\partial t}(t, x)+\nabla \cdot(\omega(t, x)(\mathbf{K} * \omega)(t, x)) & =\nu \Delta \omega(t, x), t>0, x \in \mathcal{O}, \\
\mathcal{U}(x, t) & =(\mathbf{K} * \omega)(x, t), t \geq 0, x \in \mathcal{O}, \\
\omega(0, x) & =\left(\nabla \times \mathcal{U}_{0}\right)(x), x \in \mathcal{O} \\
\mathcal{U}(t, x) & =0, t \geq 0, x \in \partial \mathcal{O}
\end{aligned}
$$

Let $\eta(x)$ and $\tau(x)$ be respectively, the outer unit normal and the unit tangent to $\partial \mathcal{O}$ at the point $x$. As we will see, the no-flow boundary condition

$$
\mathcal{U}(t, x) \cdot \eta(x)=0, t \geq 0, x \in \partial \mathcal{O}
$$

is straightforward to satisfy. But new difficulties arrive from the no-slip boundary condition

$$
\mathcal{U}(t, x) \cdot \tau(x)=0, t \geq 0, x \in \partial \mathcal{O}
$$

Indeed, let $G$ denotes now the Green function of $\Delta$ on $\mathcal{O}$ with homogeneous Dirichlet condition (i.e. $G(x, y)=0$ if $y \in \partial \mathcal{O}, G(x, y)=G(y, x))$. As previously, $\nabla \cdot \mathcal{U}=0$ implies the existence of a stream function $\psi$ such that $\mathcal{U}(t, x)=\nabla^{\perp} \psi$. Obviously $\psi$ is defined here up to an additive constant. Moreover, as $\mathcal{U}(t, x) \cdot \eta(x)=0$, $t \geq 0, x \in \partial \mathcal{O}, \psi$ is constant on the boundary $\partial \mathcal{O}$. Finally, $\psi$ must still solves $\Delta \psi=-\omega$ in $\mathcal{O}$. The problem is 
to reconstruct the velocity field from the vorticity. If we choose the stream function

$$
\psi(t, x)=-\int_{\mathcal{O}} G(x, y) \omega(t, y) d y
$$

then the velocity field

$$
\mathcal{U}(t, x)=-\int_{\mathcal{O}} \nabla_{x}^{\perp} G(x, y) \omega(t, y) d y
$$

satisfies the no-flow boundary condition (24), while the no-slip boundary condition (24) is not satisfied. Chorin $[12,13]$ has shown that to ensure $\mathcal{U}(t, x) \cdot \tau(x)=0$ on $\partial \mathcal{O}$, vorticity has to be created on the boundary. A probabilistic interpretation of the vortex equation (23) must take into account this phenomenon of creation of vorticity.

In [4], Benachour, Roynette and Vallois associate a nonlinear branching process to Equation (23), based on the remark that, if $\omega$ solves (23), then for any bounded Borel function $h: \overline{\mathcal{O}} \rightarrow \mathbb{R}$,

$$
\int_{\mathcal{O}} h(x) \omega(t, x) \lambda(d x)=\mathbb{E}\left[h\left(X_{t}\right) \exp \left(\int_{0}^{t} \phi_{c}\left(r, X_{r}\right) d A_{r}\right)\right]
$$

where $\lambda(d x)$ denotes the normalized Lebesgue measure on $\mathcal{O}, \phi_{c}(s, x)=\frac{1}{\omega+c}(\nabla \omega \cdot \eta)(s, x)$, the constant $c$ is such that $\omega_{0}(x)+c>0$, for all $x \in \overline{\mathcal{O}}$, and the couple of processes $(X, A)$ solves the following reflected stochastic differential equation in $\overline{\mathcal{O}}$

$$
\begin{aligned}
& X_{t}=X_{0}+B_{t}-\int_{0}^{t}\left(\nabla_{x}^{\perp} G * \omega\right)\left(r, X_{r}\right) d r-\int_{0}^{t} \eta\left(X_{r}\right) d A_{r}, t \geq 0, \\
& A_{t}=\int_{0}^{t} \mathbb{1}_{\left\{X_{r} \in \partial \mathcal{O}\right\}} d A_{r}, t \geq 0 .
\end{aligned}
$$

In some sense, the process $\left(\int_{0}^{t} \eta\left(X_{r}\right) d A_{r}, t \geq 0\right)$ is the minimal process which forces the solution $X$ to stay in $\overline{\mathcal{O}}$. The initial position $X_{0}$ is distributed according to $\frac{1}{\int_{\mathcal{O}}\left(\omega_{0}(x)+c\right) d x}\left(\omega_{0}+c\right)(x) \lambda(d x)$. If the sign of $\phi_{c}$ is constant negative, $\omega$ is the density of $X_{t}$, killed with the multiplicative functional $\left(C_{t}=\exp \left(\int_{0}^{t} \phi_{c}\left(r, X_{r}\right) d A_{r}, t \geq 0\right)\right.$. But here, $\phi_{c}$ is not signed. The authors propose a branching particle system $Y$ such that

$$
\int_{\mathcal{O}} h(x) \omega(t, x) \lambda(d x)=\mathbb{E} \int_{\mathcal{O}} h(x) \omega(t, x) d Y_{t}(x), \quad \text { with } Y_{t}=\sum_{i=1}^{N_{t}} \alpha_{i}(t) \delta_{Y_{t}^{i}} .
$$

This branching process $Y$ could be described as follows: choose an exponential random variable $\zeta_{1}$ with parameter one, independent of $X$. The dynamic of the initial particle is given by $X$ up to the first branching time $T_{1}=\inf \left\{s \geq 0 ; C_{s}>\zeta_{1}\right\}$. Due to the definition of $\left(C_{t}\right)$, at time $T_{1}$, the particle is located on the boundary $\partial \mathcal{O}$. If $(\nabla \omega \cdot \eta)\left(T_{1}, X_{T_{1}}\right)<0$, the particle dies. If $(\nabla \omega \cdot \eta)\left(T_{1}, X_{T_{1}}\right) \geq 0$, the particle dies and two particles spring from the ancestor. The new particles move as $X$ independently up to a second branching stopping time, and so on. The authors propose a particle algorithm associated with the branching process, without the corresponding propagation of chaos result. Remark that, in comparison with the vortex method for the Navier-Stokes equation in the whole space $\mathbb{R}^{2}$, this method need to approximate the processes $C_{t}^{i}$ related to each particles, which seems numerically very difficult.

More recently Jourdain and Méléard [21] propose another approach consisting in introducing directly a boundary condition in the vortex form of the Navier-Stokes equation and give the probabilistic interpretation of 
the vorticity in the presence of this additional condition. More precisely, they consider the following equation

$$
\begin{aligned}
\frac{\partial \omega}{\partial t}(t, x)+\nabla \cdot(\omega(\mathbf{K} * \omega)(t, x)) & =\nu \Delta \omega(t, x), t \in(0, T], x \in \mathcal{O}, \\
\omega(0, x) & =\left(\nabla \times \mathcal{U}_{0}\right)(x), x \in \mathcal{O}, \\
\nabla \omega \cdot \eta & =g \text { on }(0, T] \times \partial \mathcal{O} .
\end{aligned}
$$

The Neumann's boundary condition that permits to reconstruct the truth velocity field of the Navier-Stokes equation (22), involves a function $g$ which depends on the first derivatives of $\omega$ (see [15]). Jourdain and Méléard do not consider this particular nonlinearity and state the existence of the unique weak solution for a given function $g$. They define a nonlinear martingale problem even if two main differences arise with respect to the situation describe in Section 2: first the diffusion processes are reflected at the boundary and second the treatment of the Neumann's boundary condition in (26) involving the function $g$ generate space time random birth located at the boundary. They construct the particle algorithm associated to the nonlinear martingale problem and prove the convergence with a propagation of chaos result.

\subsection{Prandtl equation and the vortex sheet method}

Let us consider the Navier-Stokes equation (22) in the particular domain $\mathcal{O}=\mathbb{R} \times \mathbb{R}^{+}$. For small viscosities $\nu$, outside the boundary layer (we call boundary layer a small neighborhood of the boundary, of thickness of order $\mathcal{O}(\sqrt{\nu}))$ the Navier-Stokes equations is well approximated by the Euler equation $(\nu=0)$, governing the velocity field $\mathcal{U}^{E}(t, x, y)=\left(u^{E}(t, x, y), v^{E}(t, x, y)\right)$ :

$$
\begin{aligned}
\frac{\partial \mathcal{U}^{E}}{\partial t}(t, x, y)+\mathcal{U}^{E}(t, x, y) \cdot \nabla \mathcal{U}^{E}(t, x, y)+\nabla p^{E}(t, x, y) & =0, t \in(0, T], \quad(x, y) \in \mathcal{O}, \\
\nabla \cdot \mathcal{U}^{E} & =0, \text { in }(0, T] \times \mathcal{O}, \\
\mathcal{U}^{E}(0, x, y) & =\mathcal{U}_{0}(x, y), \text { in } \mathcal{O}, \\
v^{E}(t, x, y) & =0, \text { in }(0, T] \times \partial \mathcal{O}
\end{aligned}
$$

On the boundary layer, the effect of the viscosity is of order $O(1)$ and this is true even when the viscosity goes to zero due to the no-slip boundary condition that create vorticity at the boundary. In order to eliminate the non-significant phenomena, it is classical to make following scale change

$$
Y=\frac{y}{\varepsilon} \text { with the particular choice } \varepsilon=\nu
$$

The resulting equations governing the velocity field $\mathcal{U}^{P}(x, Y)=\left(u^{P}(x, Y), \sqrt{\nu} v^{P}(x, Y)\right)$ are the so called Prandtl equations

$$
\begin{aligned}
\frac{\partial u^{P}}{\partial t}+u^{P} \frac{\partial u^{P}}{\partial x}+v^{P} \frac{\partial u^{P}}{\partial Y}+\frac{\partial p}{\partial x} & =\frac{\partial^{2} u^{P}}{\partial Y^{2}}, \text { in }(0, T] \times \partial \mathcal{O}, \\
\frac{\partial p}{\partial Y} & =0, \text { in }(0, T] \times \mathcal{O}, \\
\frac{\partial u^{P}}{\partial x}+\frac{\partial v^{P}}{\partial Y} & =0, \text { in }(0, T] \times \mathcal{O}, \\
\lim _{Y \rightarrow \infty} u^{P}(t, x, Y) & =u^{E}(t, x, 0), \text { in }(0, T] \times \mathcal{O}, \\
u^{P}(0, x, Y) & =u_{0}(x, Y), \text { in } \mathcal{O}, \\
u^{P}(t, x, Y=0) & =0, \text { in }(0, T] \times \partial \mathcal{O} .
\end{aligned}
$$


As $\frac{\partial p}{\partial Y}=0$, the pressure can be reconstruct from the Euler equation by

$$
\frac{\partial p}{\partial x}(t, x, 0)=-\frac{\partial u^{E}}{\partial t}(t, x, 0)-u^{E}(t, x, 0) \frac{\partial u^{E}}{\partial x}(t, x, 0) .
$$

According to the scale change in the boundary layer, the vorticity is now defined by

$$
\omega^{P}(t, x, Y)=-\frac{\partial u^{P}}{\partial Y}(t, x, Y) .
$$

From $(29 \mathrm{c}, 29 \mathrm{~d})$, we reconstruct the velocity from the vorticity by

$$
\begin{aligned}
& u^{P}(t, x, Y)=u^{E}(t, x, 0)+\int_{Y}^{\infty} \omega^{P}(t, x, z) d z \\
& v^{P}(t, x, Y)=-\int_{0}^{Y} \frac{\partial u^{P}}{\partial x}(t, x, z) d z
\end{aligned}
$$

From (29a), we remark also that $\frac{\partial^{2} u^{P}}{\partial Y^{2}}=-\nabla_{(x, Y)} \omega^{P} \cdot \eta=-\frac{\partial p}{\partial x}$ on $\partial \mathcal{O}$. Then, after derivation in (29) we get the following vorticity equation

$$
\begin{aligned}
\frac{\partial \omega^{P}}{\partial t}+\nabla \cdot\left(\omega^{P} U\right) & =\frac{\partial^{2} \omega^{P}}{\partial Y^{2}}, \quad \text { in }(0, T] \times \mathcal{O}, \\
\omega^{P}(0, x, Y) & =-\frac{\partial u_{0}}{\partial y}(x, Y), \text { in } \mathcal{O}, \\
\nabla \omega^{P} \cdot \eta & =\frac{\partial p}{\partial x}, \text { in }(0, T] \times \partial \mathcal{O},
\end{aligned}
$$

where the field $U=\left(u^{P}, v^{P}\right)$ is given by (32) and the Neumann's boundary condition is given by (30).

The particle approximation of the Prandtl equation was initially proposed by Chorin [13] and is usually referred to as the vortex sheet method. This method was widely used in random vortex method computations. We describe it shortly: the vorticity at time $t=k \Delta t$ is approximated by a sum of Dirac masses

$$
\bar{\omega}(t, x, Y)=\sum_{j} \omega_{j} \phi_{l}\left(x-x_{j}(t)\right) \delta_{y_{j}(t)}(Y) .
$$

Each term of the sum is referred to as a vortex sheet. The $j$ th sheet has the center $\left(x_{j}(t), y_{j}(t)\right)$ and weight $\omega_{j}$. The most commonly used cutoff function $\phi_{l}(x)=\phi(x / l)$ is the hat function, originally proposed by Chorin

$$
\phi(x):=\left\{\begin{array}{cl}
1-|x|, & |x| \geq 1 \\
0, & \text { otherwise }
\end{array}\right.
$$

The parameter $l$ is often called the sheet length. According to (32), the approximated velocity field is determined by

$$
\bar{u}^{P}(t, x, Y):=u^{E}(t, x, 0)+\sum_{j} \omega_{j} \phi_{l}\left(x-x_{j}(t)\right) H\left(y_{j}(t)-Y\right)
$$

and approximating $\frac{\partial u^{P}}{\partial x}$ with a centered divided difference,

$$
\bar{v}^{P}(t, x, Y):=-\frac{\partial u^{E}}{\partial x}(t, x, 0) Y-\frac{1}{l} \sum_{j} \omega_{j}\left(\phi _ { l } \left(x^{+}-x_{j}(t)-\phi_{l}\left(x^{-}-x_{j}(t)\right) \min \left(Y, y_{j}(t)\right) .\right.\right.
$$


where $x^{+}=x+l / 2$ and $x^{-}=x-l / 2$. This velocity is completely determined by the sheet positions $\left(x_{j}(t), y_{j}(t)\right)$. Given $\bar{u}^{P}(t, x, Y)$ and $\bar{v}^{P}(t, x, Y)$, the positions at time $t+\Delta t$ are determined by a fractional step method. The first step is the numerical solution of the convection part of (33)

$$
\frac{\partial \omega}{\partial t}+\nabla \cdot(\omega \mathcal{U})=0
$$

which is

$$
\left(x_{j}^{\frac{1}{2}}(t+\Delta t), y_{j}^{\frac{1}{2}}(t+\Delta t)\right)=\left(x_{j}(t), y_{j}(t)\right)+\Delta t\left(\bar{u}^{P}\left(t, x_{t}(t), y_{j}(t)\right), \bar{v}^{P}\left(t, x_{t}(t), y_{j}(t)\right)\right)
$$

The second step is the numerical solution of the diffusive part of (33) subject to the no-slip boundary condition $u^{P}(t, x, 0)=0$. In general, the sheet positions $\left(x_{j}^{\frac{1}{2}}(t+\Delta t), y_{j}^{\frac{1}{2}}(t+\Delta t)\right)$ induce a non-zero tangential velocity on the boundary. In order to approximately satisfy it, new sheets are introduced on a spaced grid points $a_{r}, r=1, \ldots, M$, at $y=0$ with grid spacing of size $l$. Let $\omega_{\max }$ denotes a computational parameter. For each $r$, one creates $\left[\left|u^{P, \frac{1}{2}}\left(a_{r}, 0\right)\right| / \omega_{\max }\right]$ new sheets, centered at $\left(a_{r}, 0\right)$ with strengths $-\operatorname{sign}\left(u^{P, \frac{1}{2}}\left(a_{r}, 0\right)\right) \omega_{\max }$. The numerical solution of the diffusion equation $\frac{\partial \omega}{\partial t}=\frac{\partial^{2} \omega}{\partial Y^{2}}$ consists in letting all the sheets (new and old) undergo a random walk in the $y$ direction, symmetrized with respect to the origin, which corresponds to a discrete version of the reflected Brownian motion and which is compatible with the Neumann boundary condition $\nabla \omega \cdot \eta=\frac{\partial p}{\partial x}$ :

$$
\left(x_{j}(t+\Delta t), y_{j}(t+\Delta t)\right)=\left(x_{j}^{\frac{1}{2}}(t+\Delta t),\left|y_{j}^{\frac{1}{2}}(t+\Delta t)+g_{j}\right|\right),
$$

where the $g_{j}$ are i.i.d. Gaussian random variables with law $\mathcal{N}(0, \Delta t)$. For more details about the vortex sheets method and some partial rate of convergence result see e.g. [39].

At this time, there is no probabilistic interpretation of the Prandtl equation. The work of Jourdain and Méléard on the Navier-Stokes equation with linear Neumann boundary condition offer an interesting starting point for this problem.

\subsection{Interacting particles system in Lagrangian modeling of turbulent flows}

In this subsection, we give a brief overview of the Lagrangian modeling of turbulent flows, used by physicists and derived from the direct statistical approach of turbulent flows.

In the statistical approach, the properties of the fluid are assumed to be random fields. This means that the velocity and the pressure depend on possible realizations $\omega \in \Omega$. At a fixed $\omega,(t, x) \rightarrow \mathcal{U}(t, x, \omega)$ is a realization of the velocity field. If one able to identify the underlying probability space $(\Omega, \mathcal{F}, \mathbb{P})$, then for a fixed couple $(t, x), \omega \rightarrow \mathcal{U}(t, x, \omega)$ is a random variable. Thus, the Reynolds averages (or ensemble averages) are expectations:

$$
\langle\mathcal{U}\rangle(t, x):=\int_{\Omega} \mathcal{U}(t, x, \omega) d \mathbb{P}(\omega)
$$

The corresponding Reynolds decomposition of the velocity is

$$
\mathcal{U}(t, x, \omega)=\langle\mathcal{U}\rangle(t, x)+\mathbf{u}(t, x, \omega)
$$

where the random field $\mathbf{u}(t, x, \omega)$ is called the turbulent part of the velocity. Let us consider the incompressible Navier-Stokes equation in $\mathbb{R}^{3}$, for the velocity field $\mathcal{U}=\left(\mathcal{U}^{(1)}, \mathcal{U}^{(2)}, \mathcal{U}^{(3)}\right)$ and the pressure $\mathbf{p}$

$$
\begin{aligned}
\partial_{t} \mathcal{U}+(\mathcal{U} \cdot \nabla) \mathcal{U}+\nabla \mathbf{p} & =\nu \Delta \mathcal{U}, t>0, x=\left(x_{1}, x_{2}, x_{3}\right) \in \mathbb{R}^{3}, \\
\nabla \cdot \mathcal{U} & =\partial_{x_{1}} \mathcal{U}_{1}+\partial_{x_{2}} \mathcal{U}_{2}+\partial_{x_{3}} \mathcal{U}_{3}=0, t \geq 0, x \in \mathbb{R}^{3}, \\
\mathcal{U}(0, x) & =\mathcal{U}_{0}(x), x \in \mathbb{R}^{3} .
\end{aligned}
$$


Here, we consider a fluid with constant mass density. The corresponding Reynolds averaged equation for the mean velocity is

$$
\begin{gathered}
\partial_{t}\left\langle\mathcal{U}^{(i)}\right\rangle+\sum_{j=1}^{3}\left\langle\mathcal{U}^{(j)}\right\rangle \partial_{x_{j}}\left\langle\mathcal{U}^{(i)}\right\rangle+\sum_{j=1}^{3} \partial_{x_{j}}\left\langle\mathbf{u}^{(i)} \mathbf{u}^{(j)}\right\rangle+\partial_{x_{i}}\langle\mathbf{p}\rangle=\nu \sum_{j=1}^{3} \partial_{x_{j}^{2}}^{2}\left\langle\mathcal{U}^{(i)}\right\rangle, t \geq 0, x \in \mathbb{R}^{3}, \\
\sum_{j=1}^{3} \partial_{x_{j}}\left\langle\mathcal{U}^{(i)}\right\rangle=0, t \geq 0, x \in \mathbb{R}^{3} \\
\langle\mathcal{U}\rangle(0, x)=\left\langle\mathcal{U}_{0}(x)\right\rangle, x \in \mathbb{R}^{3} .
\end{gathered}
$$

Hence, to compute the averaged velocity, one needs to model the equation of the Reynolds stresses $\left(\left\langle\mathbf{u}^{(i)} \mathbf{u}^{(j)}\right\rangle, 1 \leq\right.$ $i \leq 3,1 \leq j \leq 3)$. A well known direct modeling of the Reynolds tensor is the so-called $k$-epsilon turbulence model. $k$ usually denotes the kinetic turbulent energy $k(t, x):=\sum_{i=1}^{3} \frac{1}{2}\left\langle\mathbf{u}^{(i)} \mathbf{u}^{(i)}\right\rangle(t, x)$ and $\varepsilon$ denotes the pseudodissipation

$$
\varepsilon(t, x):=\nu \sum_{i=1}^{3} \sum_{j=1}^{3} \partial_{x_{j}} \mathbf{u}^{(i)}(t, x) \partial_{x_{j}} \mathbf{u}^{(i)}(t, x) .
$$

An alternative approach consists in using the Eulerian probability density function to compute $\left\langle\mathcal{U}^{(i)}\right\rangle$ and $\left\langle\mathcal{U}^{(i)} \mathcal{U}^{(j)}\right\rangle$. Let $f_{E}(V ; t, x)$ be the probability density function (p.d.f.) of the random field $\mathcal{U}(t, x)$, then

$$
\begin{aligned}
\left\langle\mathcal{U}^{(i)}\right\rangle(t, x) & =\int_{\mathbb{R}^{3}} V^{(i)} f_{E}(V ; t, x) d V, \\
\left\langle\mathcal{U}^{(i)} \mathcal{U}^{(j)}\right\rangle(t, x) & =\int_{\mathbb{R}^{3}} V^{(i)} V^{(j)} f_{E}(V ; t, x) d V .
\end{aligned}
$$

Thus, compute $f_{E}$ allows to determine all the momentum of the velocity field. But the closure problem is transfered on the PDE satisfied by the probability density function $f_{E}$. In a series of papers (see e.g. [37]), Pope proposes to model the p.d.f. $f_{E}$ with a Lagrangian description of the flow.

To avoid the problem of computing $f_{E}$, the idea is to describe thought a stochastic model the Lagrangian properties of the flow whose law are linked in a certain way to the law of the Eulerian fields. Those models are referred to as Langevin models. Opposite to the Eulerian (i.e. macroscopic) description of the flow, the Lagrangian point of view captures its properties from a fluid particle. It consists in describing the flow properties with a state vector $(x, U, \Theta)$ which include particle location, particle velocity and a number of scalar variables denoted by $\Theta$ standing for any particles properties, and use a diffusion process to simulate its time rate of change. The associated SDE must be consistent with the macroscopic evolution of the fluid (in particular the averaged Navier-Stokes equation).

Let us consider the Simplified Langevin model (see [38]).

$$
\left\{\begin{aligned}
d \mathrm{X}_{t}= & \mathrm{U}_{t} d t \\
d \mathrm{U}_{t}^{i}= & {\left[-\frac{\partial\langle\mathbf{p}\rangle}{\partial x_{i}}\left(t, \mathrm{X}_{t}\right)-\left(\frac{1}{2}+\frac{3}{4} C_{0}\right) \frac{\langle\varepsilon\rangle\left(t, \mathrm{X}_{t}\right)}{k\left(t, \mathrm{X}_{t}\right)}\left(\mathrm{U}_{t}^{(i)}-\left\langle\mathcal{U}^{(i)}\right\rangle\left(t, \mathrm{X}_{t}\right)\right)\right] d t } \\
& +\sqrt{C_{0}\langle\varepsilon\rangle\left(t, \mathrm{X}_{t}\right)} d W_{t}^{(i)}, \forall i \in\{1,2,3\}
\end{aligned}\right.
$$

where $C_{0},\langle\varepsilon\rangle(t, x)$ and $k(t, x)$ are supposed to be known. The Reynolds averaging of the Eulerian velocity $\langle\mathcal{U}\rangle(t, x)$ must be recovered with the law of $(\mathrm{X}, \mathrm{U}) .\langle\mathbf{p}\rangle(t, x)$ must be recovered with the Poisson equation

$$
\nabla^{2}\langle\mathbf{p}\rangle=-\sum_{i=1}^{3} \sum_{j=1}^{3} \frac{\partial^{2}\left\langle\mathcal{U}^{(i)} \mathcal{U}^{(j)}\right\rangle}{\partial x_{i} \partial x_{j}}
$$


which guarantees that the averaged Eulerian velocity is divergence free. The Langevin model could be refined with other processes $\Theta$, like the turbulent frequency which allows to recover $k$ and $\langle\varepsilon\rangle$.

It remains to express the Reynolds averages $\left\langle\mathcal{U}^{(i)}\right\rangle(t, x)$ and $\left\langle\mathcal{U}^{(i)} \mathcal{U}^{(j)}\right\rangle(t, x)$ in term of the process (X, U) and its law. We call $f_{L}(V, x ; t)$ or more generally $f_{L}(V, \theta, x ; t)$ the probability density function of the random state variables $\mathrm{U}_{t}, \Theta_{t}, \mathrm{X}_{t} . f_{L}(V, \theta, x ; t)$ is solution of the Fokker-Planck equation associated to the Stochastic differential equation given by the Langevin model. In particular, contrary to $f_{E}, f_{L}$ satisfies a closed (nonlinear) PDE. In the case of incompressible flow with a constant mass density, the relationship between $f_{E}$ and $f_{L}$ is given by

$$
f_{E}(V, \theta ; x, t)=\frac{f_{L}(V, \theta, x ; t)}{\int_{\mathbb{R}^{3}} \int_{\mathbb{R}} f_{L}(V, \theta, x ; t) d V d \theta}
$$

This means that, for any bounded measurable function $g(u)$, defined on $\mathbb{R}^{3}$,

$$
\langle g(\mathcal{U})\rangle(t, x)=\int_{\mathbb{R}^{3}} g(V) f_{E}(V ; t, x) d V=\mathbb{E}\left(g\left(\mathrm{U}_{t}\right) / \mathrm{X}_{t}=x\right)
$$

In particular,

$$
\left\langle\mathcal{U}^{(i)}\right\rangle(t, x)=\int_{\mathbb{R}} V^{(i)} \frac{f_{L}(V, \theta, x ; t)}{\int_{\mathbb{R}^{3}} \int_{\mathbb{R}} f_{L}(U, \theta, x ; t) d U d \theta} d V=\mathbb{E}\left(\mathrm{U}_{t}^{(i)} / \mathrm{X}_{t}=x\right) .
$$

Thus, the particle fluid SDE equation (34) is a very special case of McKean stochastic differential equation and the Fokker-Planck PDE satisfied by $f_{L}$ can be viewed as a limit equation of a system of particles in weak interaction. In that case, it is straightforward to derive the associated stochastic particle method. To approximate the mean fields like $\left\langle\mathcal{U}^{(i)}\right\rangle(t, x)$, one uses usually the particle in cell technique. The computational space is divided in cells of given size. If $\mathcal{V}_{x}$ denotes the cell centered in $x$ then the approximation of $\left\langle\mathcal{U}^{(i)}\right\rangle(t, x)$ is given by

$$
\frac{C}{\operatorname{Volume}\left(\mathcal{V}_{x}\right)}\left(\frac{1}{N} \sum_{l=1}^{N} \mathrm{U}_{t}^{(i), l, N} \mathbb{1}_{\left\{\mathrm{X}_{t}^{l, N} \in \mathcal{V}_{x}\right\}}\right)
$$

See [36, Pope 91] for some numerical experiments for the model extended to inhomogeneous turbulent flows. See also the numerical experiments performed for turbulent polydispersed two-phase flows in [33, Minier\&Peirano 2001]. In this last case, the model couples the Eulerian and Lagrangian approaches.

\section{VisCOUS SCALAR CONSERVATION LAWS IN $\mathbb{R}$}

In this section we consider the following one-dimensional viscous scalar conservation law in $\mathbb{R}$ :

$$
\left\{\begin{array}{l}
\frac{\partial v}{\partial t}(t, x)=\frac{\sigma^{2}}{2} \frac{\partial^{2} v}{\partial x^{2}}(t, x)-\frac{\partial}{\partial x} A(v(t, x)),(t, x) \in(0,+\infty) \times \mathbb{R}, \\
v(0, x)=v_{0}(x), x \in \mathbb{R} .
\end{array}\right.
$$

This nonlinear PDE is not of McKean-Valsov type. However, when $A(x)=\frac{x^{2}}{2},(35)$ is a Fokker-Plank equation and admits a direct probabilistic interpretation. This means that there exists an associated nonlinear process 
$\left(X_{t}\right)$ such that the law of the random variable $X_{t}$ is $v(t, x) d x$. Indeed, in this cases, (35) is the Burgers equation

$$
\frac{\partial v}{\partial t}=\frac{\sigma^{2}}{2} \frac{\partial^{2} v}{\partial x^{2}}-v \frac{\partial v}{\partial x} \text { in } \mathbb{R}
$$

and the associated nonlinear $\operatorname{SDE}$ for $\left(X_{t}, t \geq 0\right)$ is

$$
\left\{\begin{array}{l}
X_{t}=X_{0}+\int_{0}^{t} v\left(s, X_{s}\right) d s+\sigma W_{t}, t \geq 0 \\
\forall t \geq 0, X_{t} \text { is of law } v(t, x) d x
\end{array}\right.
$$

where $W$ is a one dimensional Brownian motion. See Calderoni and Pulvirenti [11], Oelschäger [34], Sznitman [41]. From the numerical point of view, one have to remark that the associated particle method involves numerical approximation of the density by the empirical measure. It is possible to remove this numerical difficulty if one change the point of view of the probabilistic interpretation of (35) and use the following gradient approach.

\subsection{The gradient approach}

We introduce a probabilistic interpretation of the spatial gradient equation of (35) instead of (35) itself. More precisely, suppose that $A: \mathbb{R} \rightarrow \mathbb{R}$ is a $C^{1}$ function and that $v_{0}$ is a cumulative distribution function. This means that there exists a probability measure $U_{0}$ on $\mathbb{R}$, such that

$$
v_{0}(x)=U_{0}((-\infty, x])=\left(H * U_{0}\right)(x)
$$

See section 2.3 and Jourdain [19] for the extension to the case of non-constant function $v_{0}$ with bounded variation. If we set $u(t, x)=\partial_{x} v(t, x)$, then $u(t, x)$ solves

$$
\left\{\begin{array}{l}
\frac{\partial u}{\partial t}(t, x)=\frac{\sigma^{2}}{2} \frac{\partial^{2} u}{\partial x^{2}}(t, x)-\frac{\partial}{\partial x}\left[A^{\prime}(v(t, x)) u(t, x)\right], \forall(t, x) \in(0,+\infty) \times \mathbb{R} \\
\forall x \in \mathbb{R}, \lim _{t \rightarrow 0} u(t, x)=U_{0} .
\end{array}\right.
$$

We can reconstruct $v(t, x)$ from $u(t, x)$ by

$$
v(t, x)=\int_{-\infty}^{x} u(t, y) d y=H * u(t, \cdot)(x)
$$

Equation (36) is of McKean-Valsov type, with a constant diffusion coefficient $\sigma$ and an interacting kernel $b(x, y)=H(x-y)$. This kernel is not Lipschitz and the results on the probabilistic interpretation of Equation (10) and the convergence results given in Section 2 have to be extended to this particular situation.

We define a system of $N$ particles in mean field interaction by the following stochastic differential equation:

$$
\begin{aligned}
X_{t}^{i, N} & =X_{0}^{i, N}+\sigma W_{t}^{i}+\int_{0}^{t} A^{\prime}\left(\frac{1}{N} \sum_{j=1}^{N} H\left(X_{s}^{i, N}-X_{s}^{j, N}\right)\right) d s \\
& =X_{0}^{i, N}+\sigma W_{t}^{i}+\int_{0}^{t} A^{\prime}\left(H * \mu_{s}^{N}\left(X_{s}^{i, N}\right)\right) d s, \quad 1 \leq i \leq N
\end{aligned}
$$

where $\mu^{N}=\frac{1}{N} \sum_{i=1}^{N} \delta_{X^{i, N}}$ is the empirical measure of the particles and $\left(W^{1}, \ldots, W^{N}\right)$ is a $N$-dimensional Brownian motion independent of the initial variables $\left(X_{0}^{1, N}, \ldots, X_{0}^{N, N}\right)$ which are i.i.d. with law $m_{0}$. We have the following 
Proposition 4.1. [19, Jourdain 00] There exists a unique probability $P$ on $C([0,+\infty) ; \mathbb{R})$, solution to the following nonlinear martingale problem:

$$
\left\{\begin{array}{l}
P_{0}=U_{0} . \\
\forall \phi \in C_{b}^{2}(\mathbb{R}) \\
\phi\left(X_{t}\right)-\phi\left(X_{0}\right)-\int_{0}^{t}\left[\frac{\sigma^{2}}{2} \phi^{\prime \prime}\left(X_{s}\right)-A^{\prime}\left(H * P_{s}\left(X_{s}\right)\right) \phi^{\prime}\left(X_{s}\right)\right] d s \text { is a P-martingale. }
\end{array}\right.
$$

where $X$ is the canonical process on $C([0, \infty) ; \mathbb{R})$ and $\left(P_{t}, t \geq 0\right)=\left(P \circ X_{t}^{-1}, t \geq 0\right)$. Moreover, v(t,x)= $\left(H * P_{t}\right)(x)$ is the unique weak solution of the conservation law (35).

The particle systems $\left(X^{1, N}, \ldots, X^{N, N}\right)$ are $P$-chaotic; that is, for a fixed $j \in \mathbb{N}^{*}$, the law of $\left(X^{1, N}, \ldots, X^{j, N}\right)$ converges weakly to $P^{\otimes j}$ as $N \longrightarrow+\infty$.

As suggested by the propagation of chaos result, to construct an approximation of $v(t, x)$, we have to move the $N$ particles according to the system of stochastic differential equations (37). Let us details the numerical algorithm and the hypotheses under which its optimal rate of convergence is stated in [5]:

(H1) The function $A$ is of class $C^{3}$ and $\sigma>0$.

(H2) The measure $U_{0}$ is absolutely continuous with respect to the Lebesgue measure. Its density $u_{0}$ is a bounded function with a bounded first order derivative.

(H3) There exist constants $M>0, \eta \geq 0$ and $\alpha>0$ such that $\left|U_{0}\right|(x) \leq \eta \exp \left(-\alpha x^{2} / 2\right)$, when $|x|>M$. We construct a family $\left(y_{0}^{i}\right)_{1 \leq i \leq N}$ of initial positions such that the piecewise constant function

$$
\bar{V}_{0}(x)=\frac{1}{N} \sum_{i=1}^{N} H\left(x-y_{0}^{i}\right)
$$

approximates $v_{0}(x)$. For example, one can choose deterministic positions by inverting the function $v_{0}(x)$ :

$$
y_{0}^{i}= \begin{cases}\inf \left\{y ; U_{0}((-\infty, y])=\frac{i}{N}\right\}, & i=1, \ldots, N-1 \\ \inf \left\{y ; U_{0}((-\infty, y])=1-\frac{1}{2 N}\right\}, & i=N\end{cases}
$$

By construction, $\left\|v_{0}-\bar{V}_{0}\right\|_{\mathrm{L}^{\infty}(\mathbb{R})} \leq 1 / N$, and, under $(\mathrm{H} 3)$

$$
\left\|v_{0}-\bar{V}_{0}\right\|_{\mathrm{L}^{1}(\mathbb{R})} \leq C \sqrt{\log (N)} / N
$$

(see Theorem 2.7 and [9]). If $u_{0}$ has a compact support, the bound is $C / N$.

$(\mathrm{H} 2)$ states that $v_{0}(x)$ is in $C_{b}^{2}(\mathbb{R})$ and implies, combined with $(\mathrm{H} 1)$, that the weak solution $v(t, x)$ of $(35)$ is the classical one, i.e. $v(t, x)$ is a bounded function in $C^{1,2}([0, T] \times \mathbb{R} ; \mathbb{R})$

To get a simulation procedure for a trajectory of each $X^{i}$, we discretize in time. We choose $\Delta t$ and $K \in \mathbb{N}$ such that $T=\Delta t K$ and denote by $t_{k}=k \Delta t$ the discrete times, with $1 \leq k \leq K$. The Euler scheme leads to the following discrete-time system

$$
\left\{\begin{array}{l}
Y_{t_{k+1}}^{i}=Y_{t_{k}}^{i}+\sigma\left(W_{t_{k+1}}^{i}-W_{t_{k}}^{i}\right)+\Delta t A^{\prime}\left(\frac{1}{N} \sum_{j=1}^{N} H\left(Y_{t_{k}}^{i}-Y_{t_{k}}^{j}\right)\right) \\
Y_{0}^{i}=y_{0}^{i}, \quad i=1, \ldots, N
\end{array}\right.
$$

We approximate $v\left(t_{k}, x\right)$, solution of $(35)$, by the piecewise constant function

$$
\bar{V}_{t_{k}}(x)=\frac{1}{N} \sum_{i=1}^{N} H\left(x-Y_{t_{k}}^{i}\right) .
$$


We have the following theoretical convergence rate

Theorem 4.2. [5, Bossy 04] Assume (H1), (H2) and (H3). There exists a positive constant C, depending only on $V_{0}, A, \sigma$ and $T$, such that for all $k$ in $\{1, \ldots, K\}$,

$$
\begin{aligned}
& \sup _{x \in \mathbb{R}} \mathbb{E}\left|v\left(t_{k}, x\right)-\bar{V}_{t_{k}}(x)\right| \leq C\left(\left\|v_{0}-\bar{V}_{0}\right\|_{\mathrm{L}^{\infty}(\mathbb{R})}+\frac{1}{\sqrt{N}}+\Delta t\right) \\
\text { and } \quad & \mathbb{E}\left\|V\left(t_{k}, \cdot\right)-\bar{V}_{t_{k}}(\cdot)\right\|_{\mathrm{L}^{1}(\mathbb{R})} \leq C\left(\left\|V_{0}-\bar{V}_{0}\right\|_{\mathrm{L}^{1}(\mathbb{R})}+\frac{1}{\sqrt{N}}+\Delta t\right) .
\end{aligned}
$$

We should mention that the algorithm and its rate of convergence result could be extended to a larger class of initial data by considering $v_{0}$ as the distribution function of a signed and finite measure. Typically, $v_{0}(x)=\beta+H * U_{0}(x)$, where $U_{0} \neq 0$ is a signed and bounded measure and $\beta$ is a constant. Instead of identical weights equal to $1 / N$, the particles should have signed weights, fixed at time 0 and chosen according to the signed initial measure $U_{0}$. See [19], for the probabilistic interpretation of (35) in this particular case and [9] for a description of the algorithm using signed weights for the Burgers equation.

\subsection{Numerical comparison of performance}

Stochastic particle methods and classical deterministic methods for PDEs are very different in their principles and properties. In [6], one can found an example of detailed comparison of numerical performance on the simple model of the Burgers equation

$$
\frac{\partial v}{\partial t}(t, x)=\nu \frac{\partial^{2} v}{\partial x^{2}}(t, x)-v(t, x) \frac{\partial v}{\partial x}(t, x),(t, x) \in(0, T) \times \mathbb{R} .
$$

The particle method uses the gradient method presented in the previous section with $A(x)=\frac{x^{2}}{2}$ and $\sigma=\sqrt{2 \nu}$. The deterministic method is based upon a weak formulation of the Burgers equation seen as a conservation law satisfied on each part of the computational domain called cell or finite volume. Convection terms and diffusion terms are treated apart, to take into account the specific character of the nonlinear hyperbolic operator, and just erase the diffusion terms in case of pure convection. In this section, we summarize the main characteristics and conclusions of these comparisons.

For each method, accuracy (measured by the difference with an exact solution), computational time and storage are quantities defining the global efficiency of the method. Here, only the computational time (CPU time) is considered. In the following numerical tests, the simulation parameters have been tuned in order to require a computational time of one, five or ten minutes, on the same computer, with similarly optimized FORTRAN programs.

For comparison criteria, the error estimate is the $\mathrm{L}^{1}(\mathbb{R})$ norm of the difference between the exact and approximate solutions. For the stochastic method, this choice is natural since convergence theorems are formulated for an $\mathrm{L}^{1}(\mathbb{R})$ error. For the finite volume deterministic method, any $\mathrm{L}^{p}(\mathbb{R})$ error, $p<\infty$, is reasonable.

\subsubsection{An unstationary viscous case}

We consider exact solutions of the viscous Burgers equation, for which the accurate computation of point values is possible for a wider range of the viscosity $\nu$ :

$$
v(t, x)=10-\tanh \left(\frac{x-10 t}{2 \nu}\right) .
$$

This unsteady solution looks like a viscous shock propagating at the speed 10. Burgers equation is timeintegrated till $t=10$ and the shock goes over the interval [0;100] (see Figure 1). Figure 2 and Table 1 clearly show the difference of behaviors between the two methods with respect to the viscosity. When the viscosity is small, the deterministic method is less accurate since the exact solution has large gradients. Conversely, the 


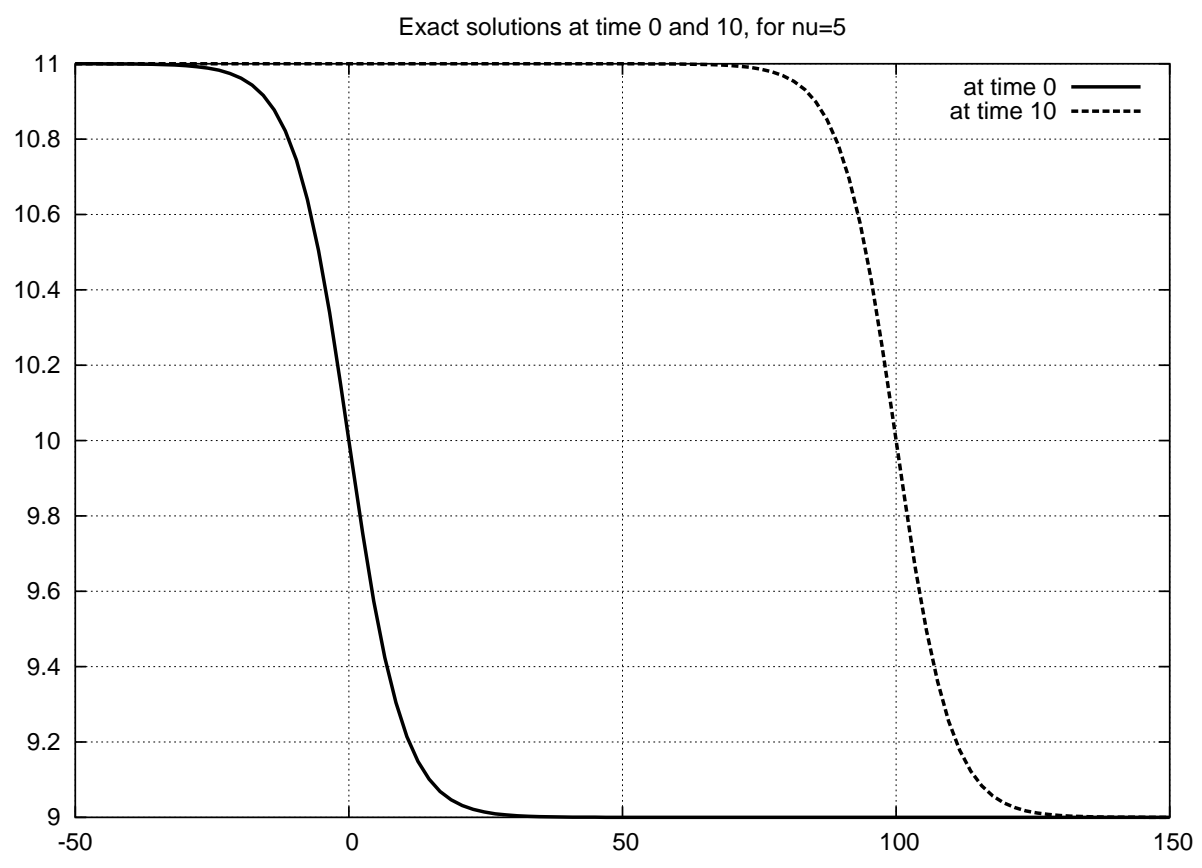

Figure 1. The exact solutions $v(t, x)$ given by $(42)$.

stochastic method is much more precise when the viscosity is small since the statistical error decreases with the viscosity.

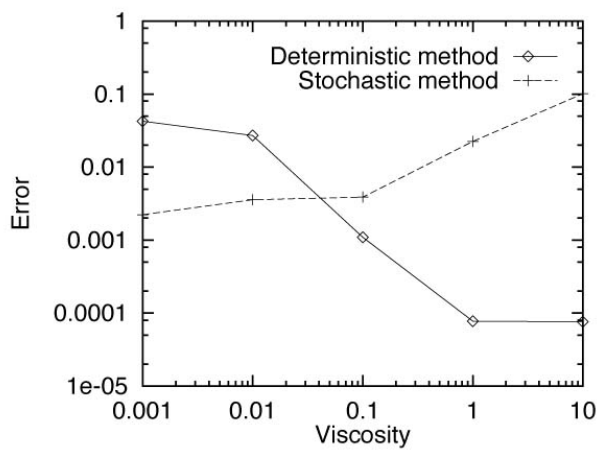

Figure 2. Errors for the stochastic and deterministic methods solving (42), for different values of the viscosity $\nu$ (CPU time is $5 \mathrm{~min}$ ). 


\begin{tabular}{|c||l||c||c|}
\hline \hline$\nu$ & CPU time & $\begin{array}{c}\text { Stochastic } \\
\text { Method }\end{array}$ & $\begin{array}{c}\text { Deterministic } \\
\text { Method }\end{array}$ \\
\hline 0.001 & $1 \mathrm{~min}$ & $2.74 .10^{-3}$ & 0.1 \\
\hline & $10 \mathrm{~min}$ & $1.64 .10^{-3}$ & $2.61 .10^{-2}$ \\
\hline \hline 0.01 & $1 \mathrm{~min}$ & $4.36 .10^{-3}$ & $8.49 .10^{-2}$ \\
\hline & $10 \mathrm{~min}$ & $3.12 .10^{-3}$ & $1.63 .10^{-2}$ \\
\hline \hline 0.1 & $1 \mathrm{~min}$ & $1.91 .10^{-2}$ & $1.22 .10^{-2}$ \\
\hline & $10 \mathrm{~min}$ & $3.33 .10^{-3}$ & $4.37 .10^{-4}$ \\
\hline \hline 1 & $1 \mathrm{~min}$ & $4.15 .10^{-2}$ & $2.53 .10^{-4}$ \\
\hline & $10 \mathrm{~min}$ & $1.22 .10^{-2}$ & $5.42 .10^{-5}$ \\
\hline \hline 10 & $1 \mathrm{~min}$ & $0.15^{-2}$ & $2.00 .10^{-4}$ \\
\hline & $10 \mathrm{~min}$ & $6.72 .10^{-2}$ & $4.80 .10^{-5}$ \\
\hline \hline
\end{tabular}

TABLE 1. Errors for the stochastic and deterministic methods solving (42)

\begin{tabular}{|c||c||c|}
\hline \hline CPU time & Stochastic Meth. & Deterministic Meth. \\
\hline $1 \mathrm{~min}$ & $2.80 .10^{-3}$ & $6.13 .10^{-4}$ \\
\hline $5 \mathrm{~min}$ & $7.10 .10^{-4}$ & $2.73 .10^{-4}$ \\
\hline $10 \mathrm{~min}$ & $4.90 .10^{-4}$ & $2.37 .10^{-4}$ \\
\hline \hline
\end{tabular}

TABLE 2. Errors for the first inviscid case (44)

\subsubsection{Inviscid case}

The good behavior of the stochastic method when the viscosity becomes smaller, let us expect that the method could also be used for the inviscid scalar conservation law

$$
\left\{\begin{array}{l}
\frac{\partial v}{\partial t}(t, x)=-\frac{\partial}{\partial x} A(v(t, x)),(t, x) \in(0,+\infty) \times \mathbb{R} \\
v(0, x)=H * U_{0}(x), x \in \mathbb{R} .
\end{array}\right.
$$

Indeed, let $\mu^{N, \nu}$ be the empirical measure of the $N$-numerical particles, computed with the viscosity $\nu$. ( $H *$ $\left.\mu^{N, \nu}\right)(x)$ is the particle approximation of the corresponding viscous conservation law (35). Jourdain [20] shows that, if $\left(\nu_{N}\right)_{N}$ is a sequence of positive numbers such that $\lim _{N \rightarrow+\infty} \nu_{N}=0$, then the approximation $(H *$ $\left.\mu^{N, \nu_{N}}\right)(x)$ converges to the unique entropy solution $v(t, x)$ of $(43)$. The convergence of the particles system, in the limit case $\nu=0$, called sticky particles, have been studied by Brenier and Grenier in [10].

Numerical comparisons of performance with the inviscid Burgers equation, have been performed first on a simple rarefaction wave with the initial data $v_{0}(x)=\mathbb{1}_{\{x>0\}}-\mathbb{1}_{\{x<0\}}$. The unique bounded entropic solution is

$$
v(x, t)=\left\{\begin{array}{cl}
-1 & \text { if } x<-t \\
x / t & \text { if }-t<x<t \\
1 & \text { if } t<x
\end{array}\right.
$$

The second test case is a combination of shocks and rarefaction waves

$$
v_{0}(x)=\left\{\begin{array}{cl}
1 & \text { if }-3<x<-2 \\
-1 & \text { if } 2<x<3 \\
0 & \text { otherwise }
\end{array}\right.
$$




\begin{tabular}{|c||c||c|}
\hline \hline CPU time & Stochastic Meth. & Deterministic Meth. \\
\hline $1 \mathrm{~min}$ & $9.20 .10^{-4}$ & $5.88 .10^{-3}$ \\
\hline $5 \mathrm{~min}$ & $5.80 .10^{-4}$ & $3.01 .10^{-3}$ \\
\hline $10 \mathrm{~min}$ & $4.77 .10^{-4}$ & $2.15 .10^{-3}$ \\
\hline \hline
\end{tabular}

TABLE 3. Errors for the second inviscid case (46)

The unique bounded entropic solution is

$$
v(x, t)=\left\{\begin{array}{cl}
-1 & \text { if } 2-t / 2<x<3-t \\
(x-3) / t & \text { if } \max (0,3-t, 3-\sqrt{2 t})<x<3 \\
0 & \text { otherwise and } x>0 \\
-u(-x, t) & \text { if } x<0
\end{array}\right.
$$

The results obtained by the deterministic method and the stochastic method are similar (see Tables 2 and 3). However, the two test cases (44) and (46) penalize differently the two methods. The problem of the approximation of the stationary shock in the inviscid test (44) underlines the noise produced by the stochastic algorithm. The most important effect is the spreading of the shock on a little neighborhood of 0 which could be controlled by the artificial viscosity. The compensation of weights (due to a non monotonic initial condition) strengthen this effect. On the other hand, in the inviscid case (46) the size of the integration domain penalize the deterministic method, whereas the solution is smooth.

\subsection{Smoothness of the error with respect to the time step and Romberg extrapolation}

In the convergence theorem 4.2 , the analysis of the algorithm with respect to the time step $\Delta t$ is based upon the analysis of the weak convergence of the Euler scheme. The techniques applied let us expect that it is possible to expand the discretization error in powers of the discretization step size $\Delta t$ at least up to the order two.

In the case of linear stochastic differential equations in the sense of McKean, such an expansion was initially showed by Talay and Tubaro [44]. The expansion up to the order two permits to justify the use of the Romberg extrapolation which provide a second order accuracy with respect to the time step $\Delta t$.

Here, we simulated a nonlinear stochastic differential equation. The nonlinearity of the SDE implies the simulation of a particle system. Even in this nonlinear case, it must be possible to adapt the Romberg extrapolation as a speed up procedure.

Figures 3 and 4 present numerical experiments on the Burgers equation (41). We compare the numerical solution obtained with the present version of the particle method (for a given time step $\Delta t$ ) and a solution obtained by extrapolation between the solutions computed for the time steps $\Delta t$ and $\Delta t / 2$. More precisely, for a given $\Delta t$, let $\left(Y_{t_{k}}^{i, \Delta t}, i=1, \ldots, N ; k=0, \ldots, K\right)$ be the family of discrete time processes involved in the algorithm and defined in (39). We denote by $\bar{V}_{t_{k}}^{\Delta t}(x)$ the corresponding numerical solution defined in (40). For the final time $T=K \Delta t$, we define the extrapolated solution $\bar{V}_{T}^{\Delta t, \Delta t / 2}(x)$ by

$$
\bar{V}_{T}^{\Delta t, \Delta t / 2}(x)=2 \bar{V}_{T}^{\Delta t / 2}(x)-\bar{V}_{T}^{\Delta t}(x) .
$$

If we are able to expand the error as

$$
\bar{V}_{T}^{\Delta t}(x)-V(T, x)=C(x) \Delta t+\mathcal{O}\left(\Delta t^{2}\right)+R(\omega)
$$

for a small enough $\Delta t$, then we will be in a position to conclude that $\mathbb{E}\left\|\bar{V}_{T}^{\Delta t, \Delta t / 2}(x)-V(T, x)\right\|$ is of order $\mathcal{O}\left(\Delta t^{2}+1 / \sqrt{N}\right)$. The constant $C(x)$ should not depend on $\Delta t$ and the random variable $R$ should be such that $\mathbb{E}\|R\| \leq C^{\prime} / \sqrt{N}$, for an appropriate choice of the norm \|\| . Numerical tests for this extrapolation method gives 
encouraging results, even if one can observe strong local error when one increase the time step $\Delta t$ (see Figures 3 and 4 ). The sensibility on $\Delta t$ varies according to the choice of the initial condition and the diffusion parameter $\sigma$. This phenomenon can be easily explained. The constants in the expansion (48) must depend on the space variable $x$ and also on the derivatives of the solution $V$. This means that we need to choose $\Delta t$ sufficiently small to have $C(x) \Delta t$ large enough compared to $\left(\Delta t^{2}\right)$ for all $x$ and to take benefit of the extrapolation procedure at all point $x$. In a recent work, Bossy, Kohatsu-Higa and Talay show that

$$
\begin{aligned}
& \sup _{x \in \mathbb{R}} \mathbb{E}\left|V(t, x)-\left(2 \bar{V}^{\Delta t / 2, N}(t, x)-\bar{V}^{\Delta t, N}(t, x)\right)\right| \\
& +\mathbb{E}\left\|V(t, \cdot)-\left(2 \bar{V}^{\Delta t / 2, N}(t, \cdot)-\bar{V}^{\Delta t, N}(t, \cdot)\right)\right\|_{\mathrm{L}^{1}(\mathbb{R})} \\
& \leq C\left(\Delta t^{2}+\frac{1}{\sqrt{N}}+\left\|V_{0}-\bar{V}_{0}\right\|_{\mathrm{L}^{1}, \mathrm{~L}^{\infty}(\mathbb{R})}\right) .
\end{aligned}
$$

The direct extrapolation procedure does not conserve the mass of the initial measure. In particular, if the solution $V(T, x)$ is the distribution function of a probability measure, the same is true for the numerical solutions $\bar{V}_{T}^{\Delta t}(x)$ and $\bar{V}_{T}^{\Delta t / 2}(x)$ but not for $\bar{V}_{T}^{\Delta t, \Delta t / 2}(x)$. Some variants of the direct extrapolation method should be explore in order to avoid these phenomena. A tentative in this direction could be the use of the extrapolation procedure during the computation, in order to correct the drifts of the particles, and not just at the final time.

\section{Viscous SCALAR CONSERVATION LAW IN A BOUNDED INTERVAL}

We consider the following viscous scalar conservation law with non homogeneous Dirichlet boundary conditions on the interval $[0,1]$ :

$$
\left\{\begin{array}{l}
\frac{\partial}{\partial t} v(t, x)=\frac{\sigma^{2}}{2} \frac{\partial^{2}}{\partial x^{2}} v(t, x)-\frac{\partial}{\partial x} A(v(t, x)), \forall(t, x) \in(0,+\infty) \times(0,1) \\
v(0, x)=v_{0}(x), \forall x \in[0,1] \\
v(t, 0)=0 \text { and } v(t, 1)=1 .
\end{array}\right.
$$

For the probabilistic interpretation, we follow [8]. We suppose that $A: \mathbb{R} \longrightarrow \mathbb{R}$ is a $C^{1}$ function and that the initial data $v_{0}$ is the cumulative distribution function of a probability measure $U_{0}$ on $[0,1]$,

$$
\forall x \in[0,1], v_{0}(x)=U_{0}([0, x])=\left(H * U_{0}\right)(x)
$$

To take into account the Dirichlet boundary conditions, we work with a diffusion process with reflection: we introduce $(X, K)$ the canonical process on the sample path space $\mathcal{C}=C([0,+\infty) ;[0,1]) \times C([0,+\infty) ; \mathbb{R})($ endowed with the topology of uniform convergence on compact sets). For $P$ in $\mathcal{P}(\mathcal{C})$, the set of probability measures on $\mathcal{C},\left(\bar{P}_{t}, t \geq 0\right)$ denotes the set of time-marginals of the probability measure $\bar{P}$ on $C([0,+\infty) ;[0,1])$ defined by $\bar{P}=P \circ X^{-1}$. Equation (49) is then associated to the following nonlinear martingale problem

Definition 5.1. A probability measure $P \in \mathcal{P}(\mathcal{C})$ solves the martingale problem $(M P)$ starting at $U_{0} \otimes \delta_{0} \in$ $\mathcal{P}([0,1] \times \mathbb{R})$, if

(i) $P \circ\left(X_{0}, K_{0}\right)^{-1}=U_{0} \otimes \delta_{0}$.

(ii) $\forall \varphi \in C_{b}^{2}(\mathbb{R})$,

$\varphi\left(X_{t}-K_{t}\right)-\varphi\left(X_{0}-K_{0}\right)-\int_{0}^{t}\left(\frac{\sigma^{2}}{2} \varphi^{\prime \prime}\left(X_{s}-K_{s}\right)+A^{\prime}\left(H * \bar{P}_{s}\left(X_{s}\right)\right) \varphi^{\prime}\left(X_{s}-K_{s}\right)\right) d s$ is a $P$ martingale.

(iii) $P$ a.s., $\forall t \geq 0, \int_{0}^{t} d|K|_{s}<+\infty,|K|_{t}=\int_{0}^{t} 1_{\{0,1\}}\left(X_{s}\right) d|K|_{s}$ and $K_{t}=\int_{0}^{t}\left(1-2 X_{s}\right) d|K|_{s}$. 

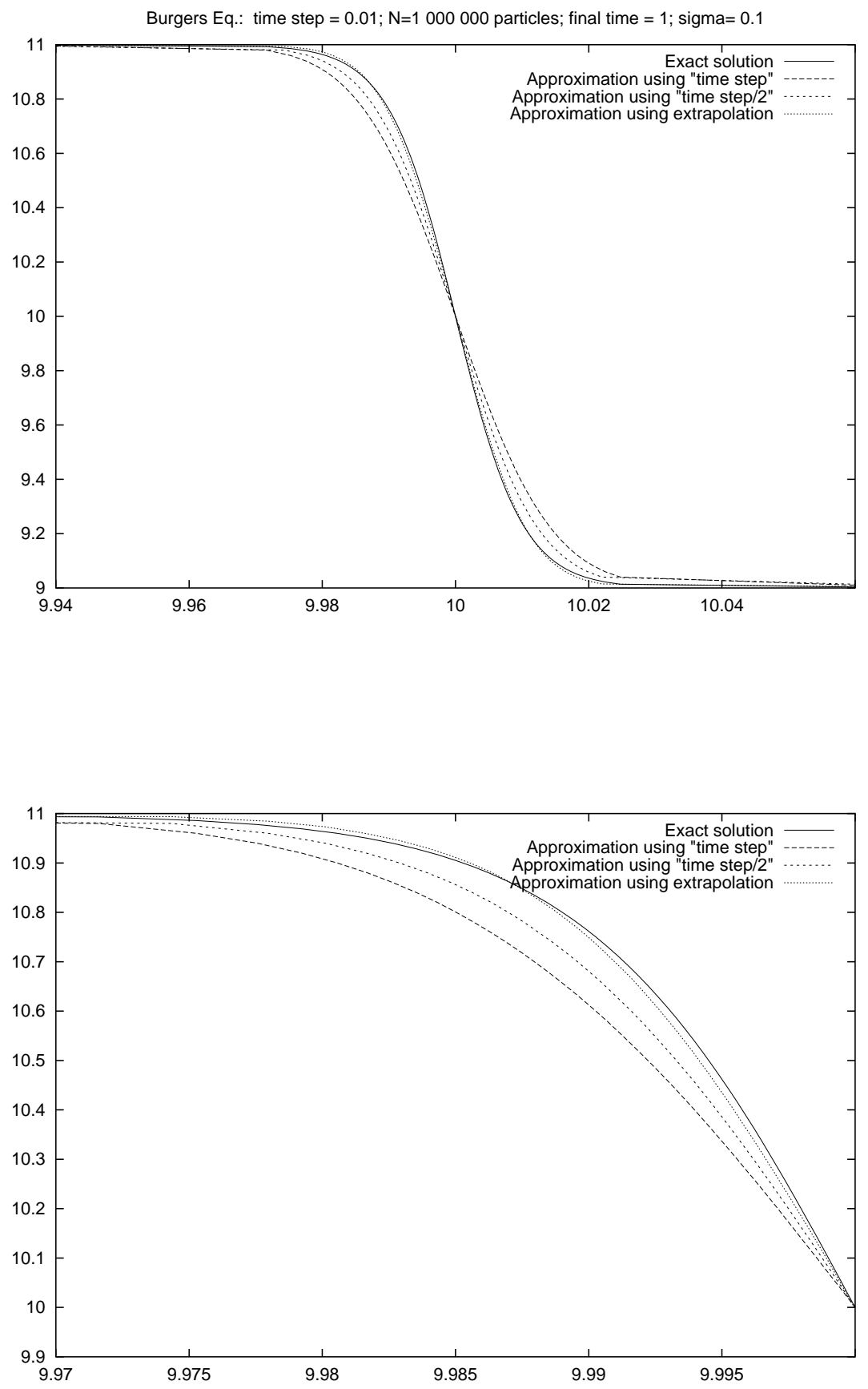

Figure 3. Exact and numerical solutions of the Burgers equation with initial condition $V(0, x)=10-\tanh \left(\frac{x}{\sigma^{2}}\right)$. The corresponding errors in the $\mathrm{L}^{1}$-norm are $\left\|V(1, x)-\bar{V}_{1}^{\Delta t}(x)\right\|_{\mathrm{L}^{1}(\mathbb{R})}=$ $0.0049,\left\|V(1, x)-\bar{V}_{1}^{\Delta t / 2}(x)\right\|_{\mathrm{L}^{1}(\mathbb{R})}=0.0024$ and $\left\|V(1, x)-2 \bar{V}_{1}^{\Delta t / 2}(x)+\bar{V}_{1}^{\Delta t}(x)\right\|_{\mathrm{L}^{1}(\mathbb{R})}=0.00062$. 

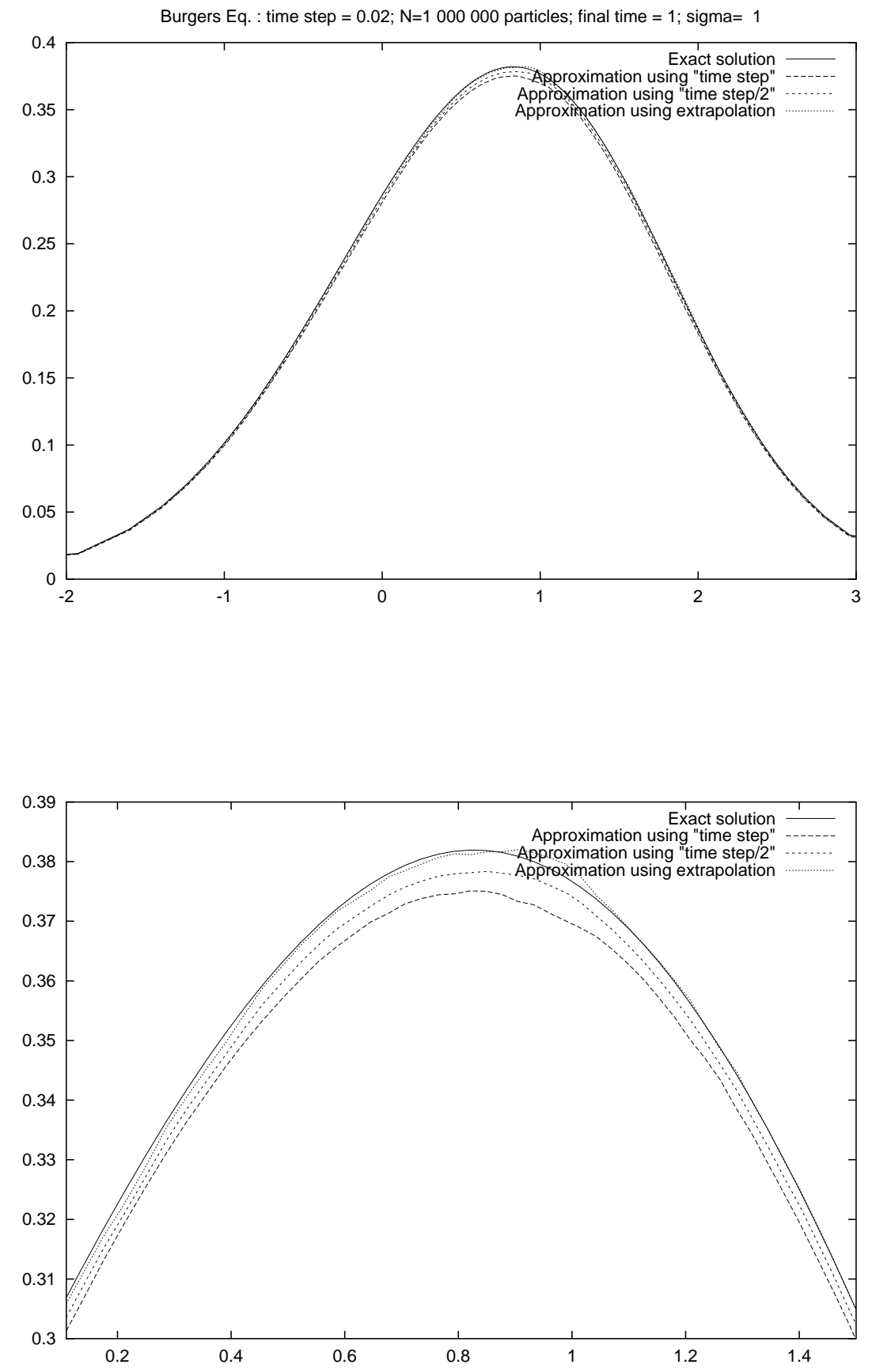

FIGURE 4. Exact and numerical solutions of the Burgers equation with initial condition $V(0, x)=H(x)-H(x-1)$. The corresponding errors in the $\mathrm{L}^{1}$-norm are $\| V(1, x)-$ $\bar{V}_{1}^{\Delta t}(x)\left\|_{\mathrm{L}^{1}(\mathbb{R})}=0.0183,\right\| V(1, x)-\bar{V}_{1}^{\Delta t / 2}(x)\left\|_{\mathrm{L}^{1}(\mathbb{R})}=0.0094,\right\| V(1, x)-2 \bar{V}_{1}^{\Delta t / 2}(x)+$ $\bar{V}_{1}^{\Delta t}(x) \|_{\mathrm{L}^{1}(\mathbb{R})}=0.0030$. 
The finite variation process $K_{t}$ which increases when $X_{t}=0$ and decreases when $X_{t}=1$ accounts for reflection and prevents $X_{t}$ from leaving the interval $[0,1]$. In other words, we look for a weak solution of the following nonlinear reflected SDE

$$
\left\{\begin{array}{l}
X_{t}=X_{0}+\int_{0}^{t}\left(A^{\prime}\left(\int_{\mathbb{R}^{d}} H\left(X_{s}-y\right) \bar{P}_{s}(d y)\right)\right) d s+\sigma W_{t}+K_{t}, \quad t \in[0, T] \\
\text { where } \bar{P}_{t}=\operatorname{Law}\left(X_{t}\right) \text { and } \\
|K|_{t}=\int_{0}^{t} \mathbb{l}_{\{0,1\}}\left(X_{s}\right) d|K|_{s}, K_{t}=\int_{0}^{t}\left(1-2 X_{s}\right) d|K|_{s} \\
X_{0} \text { of law } U_{0},
\end{array}\right.
$$

$W$ is a Brownian motion in $\mathbb{R}$, independent of $X_{0}$. We summarize the steps for solving problem $(M P)$.

- First, check that when $P$ solves $(M P),(t, x) \rightarrow\left(H * \bar{P}_{t}\right)(x)$ is the unique weak solution of (49). Uniqueness for the martingale problem follows.

- Second, existence is obtained thanks to a propagation of chaos result for a system of weakly interacting diffusion processes.

\subsection{Weak solution}

For $T>0$, let $\mathcal{Q}_{T}=(0, T) \times(0,1)$ and $W_{2}^{0,1}\left(\mathcal{Q}_{T}\right), W_{2}^{1,1}\left(\mathcal{Q}_{T}\right)$ denote the Hilbert spaces with respective scalar products

$$
\begin{aligned}
(u, v) W_{2}^{0,1}\left(\mathcal{Q}_{T}\right) & =\int_{\mathcal{Q}_{T}}\left(u v+\partial_{x} u \partial_{x} v\right) d x d t \\
{ }^{(u, v)} W_{2}^{1,1}\left(\mathcal{Q}_{T}\right) & =\int_{\mathcal{Q}_{T}}\left(u v+\partial_{x} u \partial_{x} v+\partial_{t} u \partial_{t} v\right) d x d t
\end{aligned}
$$

We introduce the Banach space $V_{2}^{0,1}\left(\mathcal{Q}_{T}\right)=\left\{u \in W_{2}^{0,1}\left(\mathcal{Q}_{T}\right) \cap C\left((0, T) ; \mathrm{L}^{2}(0,1)\right)\right.$ such that $\|u\|_{V_{2}^{0,1}\left(\mathcal{Q}_{T}\right)}=$ $\left.\sup _{0 \leq t \leq T}\|u(t, x)\|_{\mathrm{L}^{2}(0,1)}+\left\|\partial_{x} u\right\|_{\mathrm{L}^{2}\left(\mathcal{Q}_{T}\right)}<+\infty\right\}$. The corresponding subspaces consisting in elements which vanish on $[0, T] \times\{0,1\}$ are respectively denoted by $\stackrel{\circ}{W}_{2}^{0,1}\left(\mathcal{Q}_{T}\right), \stackrel{\circ}{W_{2}^{1,1}}\left(\mathcal{Q}_{T}\right), \stackrel{\circ 0,1}{V_{2}}\left(\mathcal{Q}_{T}\right)$.

Lemma 5.2. Equation (49) has no more than one weak solution in the following sense: a weak solution of (49) is a function $v:[0,+\infty) \times[0,1] \rightarrow \mathbb{R}$ satisfying the boundary conditions and such that for any $T>0$, $v \in V_{2}^{0,1}\left(\mathcal{Q}_{T}\right) \cap \mathrm{L}^{\infty}\left(\mathcal{Q}_{T}\right)$ and for all $\phi$ in $\stackrel{\circ}{W_{2}^{1,1}}\left(\mathcal{Q}_{T}\right)$ and all $t \in[0, T]$,

$$
\begin{aligned}
\int_{0}^{1} v(t, x) \phi(t, x) d x= & \int_{0}^{1} v_{0}(x) \phi(0, x) d x+\int_{0}^{t} \int_{0}^{1} \frac{\partial}{\partial s} \phi(s, x) v(s, x) d x d s \\
& +\int_{0}^{t} \int_{0}^{1} \frac{\partial}{\partial x} \phi(s, x) A(v(s, x)) d x d s \\
& -\int_{0}^{t} \int_{0}^{1} \frac{\sigma^{2}}{2} \frac{\partial}{\partial x} \phi(s, x) \frac{\partial}{\partial x} v(s, x) d x d s
\end{aligned}
$$

The proof of this lemma does not use probabilistic tools, but adapts techniques used to establish the energy inequality for generalized solution in the sense of Ladyzenskaja, Solonnikov and Ural'ceva [24] of a linear equation with uniformly bounded coefficients.

Proposition 5.3. If $P$ solves the martingale problem (MP) starting at $U_{0} \otimes \delta_{0}$, then

(i) for any $t>0, \bar{P}_{t}$ has a density $\bar{p}_{t}$ which belongs to $\mathrm{L}^{2}([0,1])$ and it holds that

$$
\left\|\bar{p}_{t}\right\|_{\mathrm{L}^{2}([0,1])} \leq C\left(1+t^{-1 / 4}\right) \exp (C t)
$$


(ii) $V(t, x)=H * \bar{P}_{t}(x)$ is a weak solution of (49). Moreover, uniqueness holds for the martingale problem $(M P)$.

Sketch of the proof. Clearly $V(t, x)=H * \bar{P}_{t}(x)$ is bounded by 1 . Let $T>0$. Thanks to (i), $V(t, x)$ belongs to $V_{2}^{0,1}\left(\mathcal{Q}_{T}\right)$ and satisfies the boundary conditions in $(49)$. Now, let $\phi$ be a $C^{\infty}$ function on $[0, T] \times[0,1]$ with $\phi(t, 0)=\phi(t, 1)=0$ for all $t \in[0, T]$ and set $\psi(t, x)=\int_{0}^{x} \phi(t, y) d y$. According to Definition 5.1 (ii), under $P$, $\frac{1}{\sigma}\left(X_{t}-K_{t}-\int_{0}^{t} A^{\prime}\left(V\left(s, X_{s}\right)\right) d s\right)$ is a local martingale with quadratic variation $t$ i.e. a Brownian motion. Thus, by Itô's formula, as $\int_{0}^{t} \frac{\partial \psi}{\partial x}\left(s, X_{s}\right) d K_{s}=\int_{0}^{t} \phi\left(s, X_{s}\right) 1_{\{0,1\}}\left(X_{s}\right) d K_{s}=0$,

$$
\mathbb{E} \psi\left(t, X_{t}\right)=\mathbb{E} \psi\left(0, X_{0}\right)+\mathbb{E} \int_{0}^{t}\left[\frac{\partial \psi}{\partial s}+\frac{\sigma^{2}}{2} \frac{\partial^{2} \psi}{\partial x^{2}}\right]\left(s, X_{s}\right) d s+\mathbb{E} \int_{0}^{t} \frac{\partial \psi}{\partial x}\left(s, X_{s}\right) A^{\prime}\left(V\left(s, X_{s}\right)\right) d s
$$

As $\bar{p}_{s}(x) d x=\frac{\partial V}{\partial x}(s, x) d x$ is the law of $X_{s}$,

$$
\begin{aligned}
\int_{0}^{1} \psi(t, x) \frac{\partial V}{\partial x}(t, x) d x= & \int_{0}^{1} \psi(0, x) \frac{\partial V_{0}}{\partial x}(x) d x+\int_{0}^{t} \int_{0}^{1} \frac{\partial \psi}{\partial s}(s, x) \frac{\partial V}{\partial x}(s, x) d x d s \\
& +\int_{0}^{t} \int_{0}^{1} \frac{\sigma^{2}}{2} \frac{\partial \phi}{\partial x}(s, x) \frac{\partial V}{\partial x}(s, x) d x d s \\
& +\int_{0}^{t} \int_{0}^{1} \phi(s, x) A^{\prime}(V(s, x)) \frac{\partial V}{\partial x}(s, x) d x d s .
\end{aligned}
$$

(50) follows, for $\phi$ a $C^{\infty}$ function vanishing $x=0$ and $x=1$. As $V$ is in $V_{2}^{0,1}\left(\mathcal{Q}_{T}\right)$, the identity is extended by density for any function $\phi$ in $\stackrel{\circ}{W_{2}^{1,1}}\left(\mathcal{Q}_{T}\right)$.

Uniqueness for $(M P)$ is derived from the uniqueness result for the problem (49): if $P$ and $Q$ solve $(M P)$, then for any $(t, x) \in[0,+\infty) \times \mathbb{R},\left(H * \bar{P}_{t}\right)(x)=\left(H * \bar{Q}_{t}\right)(x)$. Hence $P$ and $Q$ solve a linear martingale problem with bounded drift term $A^{\prime}\left(H * \bar{P}_{t}(x)\right)$ and by Girsanov theorem, $P=Q$.

The proof of (i) adapts the proof of Proposition 1.1 of Méléard and Roelly [32], to the case of reflected diffusion processes. According to Definition 5.1 (ii), under $P, \frac{1}{\sigma}\left(X_{t}-K_{t}-\int_{0}^{t} A^{\prime}\left(V\left(s, X_{s}\right)\right) d s\right)$ is a Brownian motion. As $\sup _{[0,1]}\left|A^{\prime}(x)\right|<+\infty$, by Girsanov theorem, under the probability measure $Q \in \mathcal{P}(\mathcal{C})$ such that $\left.\frac{d Q}{d P}\right|_{\mathcal{F}_{t}}=\frac{1}{Z_{t}}$ where

$$
Z_{t}=\exp \left(\int_{0}^{t} \frac{1}{\sigma^{2}} A^{\prime}\left(H * \bar{P}_{s}\left(X_{s}\right)\right) d\left(X_{s}-K_{s}\right)-\frac{1}{2 \sigma^{2}} A^{\prime 2}\left(H * \bar{P}_{s}\left(X_{s}\right)\right) d s\right)
$$

$\beta_{t}=\frac{1}{\sigma}\left(X_{t}-K_{t}\right)$ is a Brownian motion starting at $\frac{1}{\sigma} X_{0}$ and $\left(X_{t}, t \geq 0\right)$ is the doubly reflected process associated with $\left(\sigma \beta_{t}, t \geq 0\right)$. For $\psi$ bounded and measurable, since $\mathbb{E}^{P}\left(\psi\left(X_{t}\right)\right)=\mathbb{E}^{Q}\left(\psi\left(X_{t}\right) Z_{t}\right)$, by Cauchy-Schwarz inequality

$$
\mathbb{E}^{P}\left(\psi\left(X_{t}\right)\right) \leq\left(\int_{0}^{1} \psi^{2}(x) u_{t}(x) d x\right)^{\frac{1}{2}} \exp \left(\frac{t}{2 \sigma^{2}} \sup _{[0,1]}\left|A^{\prime 2}(x)\right|\right)
$$

where $u_{t}(x)=\int_{0}^{1} p_{\sigma^{2} t}(z, x) U_{0}(d z)$ and

$$
p_{t}(z, x)=\frac{1}{\sqrt{2 \pi t}} \sum_{n \in \mathbb{Z}}\left(e^{-\frac{(x-z-2 n)^{2}}{2 t}}+e^{-\frac{(x+z+2 n)^{2}}{2 t}}\right)
$$


denotes the transition density of the doubly reflected Brownian motion in $[0,1]$. It is easy to check that $p_{t}(z, x) \leq \frac{2}{\sqrt{2 \pi t}}+1$ and thus,

$$
\mathbb{E}^{P}\left(\psi\left(X_{t}\right)\right) \leq C\left(1+t^{-1 / 4}\right) \exp (C t)\|\psi\|_{\mathrm{L}^{2}([0,1])}
$$

which gives the lemma.

\subsection{The propagation of chaos result and the particle method}

Consider the system of weakly interacting diffusion processes with normal reflecting boundary conditions:

$$
\left\{\begin{array}{l}
X_{t}^{i, N}=X_{0}^{i, N}+\sigma W_{t}^{i}+\int_{0}^{t} A^{\prime}\left(H * \bar{\mu}_{s}^{N}\left(X_{s}^{i, N}\right)\right) d s+K_{t}^{i, N}, i=1, \ldots, N \\
\left|K^{i, N}\right|_{t}=\int_{0}^{t} 1_{\{0,1\}}\left(X_{s}^{i, N}\right) d\left|K^{i, N}\right|_{s} \\
K_{t}^{i, N}=\int_{0}^{t}\left(1-2 X_{s}^{i, N}\right) d\left|K^{i, N}\right|_{s}
\end{array}\right.
$$

where $\bar{\mu}_{s}^{N}=\frac{1}{N} \sum_{j=1}^{N} \delta_{X_{s}^{j, N}}$ and $\left(W^{1}, \ldots, W^{N}\right)$ is a N-dimensional Brownian motion independent of the initial variables $\left(X_{0}^{1, N}, \ldots, X_{0}^{N, N}\right)$ which are i.i.d. with law $U_{0}$.

As $\sup _{[0,1]}\left|A^{\prime}(x)\right|$ is bounded, by Girsanov theorem, this equation admits a unique weak solution. Existence for problem $(M P)$ is ensured by the following propagation of chaos result:

Theorem 5.4. [8, Bossy Jourdain 02] The particle systems $\left(\left(X^{1, N}, K^{1, N}\right), \ldots,\left(X^{N, N}, K^{N, N}\right)\right)$ are $P$-chaotic where $P$ denotes the unique solution of the martingale problem $(M P)$ starting at $U_{0} \otimes \delta_{0}$ i.e. for fixed $j \in \mathbb{N}^{*}$ the law of $\left(\left(X^{1, N}, K^{1, N}\right), \ldots,\left(X^{j, N}, K^{j, N}\right)\right)$ converges weakly to $P^{\otimes j}$ as $N \rightarrow+\infty$.

Corollary 5.5. It is possible to approximate $V(t, x)=H * \bar{P}_{t}(x)$, solution of (49), thanks to the empirical cumulative distribution function $\left(H * \bar{\mu}_{t}^{N}\right)(x)$ of the particle system:

$$
\forall(t, x) \in[0,+\infty) \times[0,1], \lim _{N \rightarrow+\infty} \mathbb{E}\left|V(t, x)-H * \bar{\mu}_{t}^{N}(x)\right|=0 .
$$

Proof. For $t>0$ and $x \in[0,1]$, according to $(51)$, the function $Q \in \mathcal{P}(\mathcal{C}) \rightarrow\left|H * \bar{P}_{t}(x)-H * \bar{Q}_{t}(x)\right| \in \mathbb{R}$ is continuous at $P$. The weak convergence of the sequence $\left(\mu^{N}\right)_{N}$ to $P$ implies

$$
\lim _{N \rightarrow+\infty} \mathbb{E}\left|H * \bar{P}_{t}(x)-H * \bar{\mu}_{t}^{N}(x)\right|=\mathbb{E}^{\pi^{\infty}}\left|H * \bar{P}_{t}(x)-H * \bar{Q}_{t}(x)\right|=0 .
$$

In case $t=0$, we conclude by the Strong Law of Large Numbers.

To turn the convergence result (53) into a numerical approximation procedure, one need to discretize in time the $N$-dimensional stochastic differential equation (52). Modified Euler schemes for reflected diffusion processes exist. For example, weak convergence for the simple projection scheme and for the symmetrized scheme have been studied respectively in [14] and [7].

The full rate of convergence of the particle approximation of $V(t, x)$ is studied in [8], for the version of the Euler scheme proposed by Lépingle [26] for one-dimensional reflected SDEs in a bounded interval. This scheme mimics exactly the reflection at the boundary: choose $\Delta t>0$ and $L \in \mathbb{N}$ such that $T=L \Delta t$ and denote by $Y_{t_{l}}^{i}$ the position of the $i$-th particle $(1 \leq i \leq N)$ at the discretization time $t_{l}=l \Delta t(0 \leq l \leq L)$. The Euler-Lépingle scheme consists in setting $0<\alpha_{0}<\alpha_{1}<1$ and in generating exact reflection on the lower-boundary on $\left[t_{l}, t_{l+1}\right]$ when $Y_{t_{l}}^{i} \leq \alpha_{0}$ and exact reflection on the upper-boundary on $\left[t_{l}, t_{l+1}\right]$ when $Y_{t_{l}}^{i} \geq \alpha_{1}$. When at the end of the time-step the computed position is smaller than 0 or greater than 1 , then $Y_{t_{l+1}}^{i}$ is respectively chosen equal to 0 
or equal to 1 . At time $t_{l}$, the function $V\left(t_{l}, x\right)$ is approximated thanks to the empirical cumulative distribution function

$$
\bar{V}\left(t_{l}, x\right)=\frac{1}{N} \sum_{i=1}^{N} H\left(x-Y_{t_{l}}^{i}\right)
$$

and the positions of the $i$ th particle are given inductively by

$$
\left\{\begin{array}{l}
Y_{t_{0}}^{i}=y_{0}^{i}, \\
Y_{t}^{i}=0 \vee\left(Y_{t_{l}}^{i}+\sigma\left(W_{t}^{i}-W_{t_{l}}^{i}\right)+\left(t-t_{l}\right) A^{\prime}\left(\bar{V}\left(t_{l}, Y_{t_{l}}^{i}\right)\right)+C_{t}^{i}\right) \wedge 1, \forall 0 \leq l \leq L-1, \forall t \in\left[t_{l}, t_{l+1}\right] \\
C_{t}^{i}=\mathbb{1}_{\left\{Y_{t_{l}}^{i} \leq \alpha_{0}\right\}} \sup _{s \in\left[t_{l}, t\right]}\left(Y_{t_{l}}^{i}+\sigma\left(W_{s}^{i}-W_{t_{l}}^{i}\right)+\left(s-t_{l}\right) A^{\prime}\left(\bar{V}\left(t_{l}, Y_{t_{l}}^{i}\right)\right)\right)^{-} \\
\quad-\mathbb{l}_{\left\{Y_{t_{l}}^{i} \geq \alpha_{1}\right\}} \sup _{s \in\left[t_{l}, t\right]}\left(Y_{t_{l}}^{i}-1+\sigma\left(W_{s}^{i}-W_{t_{l}}^{i}\right)+\left(s-t_{l}\right) A^{\prime}\left(\bar{V}\left(t_{l}, Y_{t_{l}}^{i}\right)\right)\right)^{+}
\end{array}\right.
$$

Taking advantage of the one-dimensional space domain, we invert the initial cumulative distribution function $V_{0}(x)=H * U_{0}(x)$ to construct the set of initial positions of the numerical particles:

$$
y_{0}^{i}=\inf \left\{z ;\left(H * U_{0}\right)(z) \geq \frac{i}{N}\right\} \text { for } 1 \leq i \leq N
$$

Since it is possible to simulate jointly the Brownian increment $\left(W_{t_{l+1}}^{i}-W_{t_{l}}^{i}\right)$ and the corresponding $\sup _{s \in\left[t_{l}, t_{l+1}\right]}\left(W_{s}^{i}-W_{t_{l}}^{i}+\left(s-t_{l}\right) \alpha\right)$, this discretization scheme is feasible and the weak rate of convergence is of order one (see [8]). An alternative choice could be the symmetrized Euler scheme, easy to simulate and which produce a theoretical weak convergence error of order one. But it application in its particular contested have not been studied.

The optimal rate of convergence is obtained on rather strong assumptions on the initial condition $v_{0}(x)=$ $H * U_{0}(x)$, ensuring that the weak solution of (49) is a classical solution (i.e. $C^{1}$ in the time variable $t$ and $C^{2}$ in the space variable $x)$ and also that $\partial_{x} V(t, x)$ is Hölder continuous with in the time variable:

$$
(H)\left\{\begin{array}{l}
v_{0} \in C^{2+\beta}([0,1])\left(v_{0}^{\prime \prime} \text { Hölder continuous with exponent } \beta\right) \text { where } \beta \in(0,1), \\
\sigma^{2} v_{0}^{\prime \prime}(0)=2 A^{\prime}(0) v_{0}^{\prime}(0) \text { and } \sigma^{2} v_{0}^{\prime \prime}(1)=2 A^{\prime}(1) v_{0}^{\prime}(1), \\
A \text { is a } C^{3} \text { function. }
\end{array}\right.
$$

Lemma 5.6. Under $(H)$, the solution $V(t, x)=H * \bar{P}_{t}(x)$ of $(49)$ belongs to $C^{1,2}([0, T] \times[0,1])$ and $\partial_{x} V(t, x)$ is Hölder continuous with exponent $(1+\beta) / 2$ in the time variable $t$ on $[0, T] \times[0,1]$.

In order to reduce the effort needed to compute the correction terms $C_{t}^{i}$ in (54), it is interesting to let $\alpha_{0}$ and $\alpha_{1}$ depend on the time-step $\Delta t$ and converge respectively to 0 and 1 as $\Delta t \rightarrow 0$. Supposing that these convergence are not too quick, we have the following estimate for the convergence rate of the particle method:

Theorem 5.7. Under hypothesis $(H)$, if we assume that $0<\alpha_{0}(\Delta t) \leq \alpha_{1}(\Delta t)<1$ satisfy $\alpha_{0}(\Delta t) \wedge(1-$ $\left.\alpha_{1}(\Delta t)\right) \geq a \Delta t^{\gamma}$ for $0 \leq \gamma<1 / 2$ and $a>0$, then there exists a strictly positive constant $C$ depending on $A, U_{0}, T, \bar{\sigma}, a$ and $\gamma$ such that

$$
\forall 0 \leq l \leq L, \sup _{x \in[0,1]} \mathbb{E}\left|V\left(t_{l}, x\right)-\bar{V}\left(t_{l}, x\right)\right| \leq C\left(\frac{1}{\sqrt{N}}+\Delta t\right) .
$$

The proof in [8] follows the main ideas of [5, Bossy 04] who deals with the convergence rate of a particle approximation for the solution of the scalar conservation law with spatial domain $\mathbb{R}$ similar to (49). New difficulties arise in the present framework because of the reflection. An important step is the Weak error of the 
Euler-Lépingle scheme: recall that $T=L \Delta t(\Delta t>0, L \in \mathbb{N})$ and $t_{l}=l \Delta t$ for $0 \leq l \leq L$. The Euler-Lépingle discretization of the stochastic differential equation

$$
\left\{\begin{array}{l}
X_{t}^{y}=y+\sigma W_{t}+\int_{0}^{t} b\left(s, X_{s}^{y}\right) d s+K_{t}^{y} \\
\left|K^{y}\right|_{t}=\int_{0}^{t} \mathbb{1}_{\{0,1\}}\left(X_{s}^{y}\right) d\left|K^{y}\right|_{s}, K_{t}^{y}=\int_{0}^{t}\left(1-2 X_{s}^{y}\right) d\left|K^{y}\right|_{s}
\end{array}\right.
$$

is given by

$$
\left\{\begin{array}{l}
\tilde{X}_{t_{0}}^{y}=y \\
\forall t \in\left[t_{l}, t_{l+1}\right], \tilde{X}_{t}^{y}=0 \vee\left(\tilde{X}_{t_{l}}^{y}+\sigma\left(W_{t}-W_{t_{l}}\right)+b\left(t_{l}, \tilde{X}_{t_{l}}^{y}\right)\left(t-t_{l}\right)+C_{t}\right) \wedge 1 \\
C_{t}=\mathbb{I}_{\left\{\tilde{X}_{t_{l}}^{y} \leq \alpha_{0}\right\}} \sup _{s \in\left[t_{l}, t\right]}\left(\tilde{X}_{t_{l}}^{y}+\sigma\left(W_{s}-W_{t_{l}}\right)+b\left(t_{l}, \tilde{X}_{t_{l}}^{y}\right)\left(s-t_{l}\right)\right)^{-} \\
\quad-\mathbb{I}_{\left\{\tilde{X}_{t_{l}}^{y} \geq \alpha_{1}\right\}} \sup _{s \in\left[t_{l}, t\right]}\left(\tilde{X}_{t_{l}}^{y}-1+\sigma\left(W_{s}-W_{t_{l}}\right)+b\left(t_{l}, \tilde{X}_{t_{l}}^{y}\right)\left(s-t_{l}\right)\right)^{+}
\end{array}\right.
$$

Assuming a regularity condition on the drift coefficient $b(s, x)$ which is satisfied by $A^{\prime}(V(s, x))$ under hypothesis $(H)$ (see Lemma 5.6), we have the following weak convergence rate:

Proposition 5.8. [8] Assume that b is $C^{1,2}$ on $[0, T] \times[0,1]$, that for some $\alpha>0, \partial_{x} b(t, x)$ is Hölder continuous with exponent $\alpha$ in $t$ and that $0<\alpha_{0}(\Delta t) \leq \alpha_{1}(\Delta t)<1$ satisfy $\alpha_{0}(\Delta t) \wedge\left(1-\alpha_{1}(\Delta t)\right) \geq a \Delta t^{\gamma}$ for $\gamma \in[0,1 / 2)$ and $a>0$. Then there is a constant $C$ depending on $\sigma, T, b, a, \gamma$ but not on $y$ and $\Delta t$ such that when $f:[0,1] \rightarrow \mathbb{R}$ $i s$ a function with bounded variation and $m$ denotes its distribution derivative,

$$
\forall l \leq L,\left|\mathbb{E}\left(f\left(X_{t_{l}}^{y}\right)-f\left(\tilde{X}_{t_{l}}^{y}\right)\right)\right| \leq C \Delta t \int_{0}^{1}|m|(d x) .
$$

\subsection{Numerical experiments}

In this subsection, we summarize the numerical experiments in [8]. As a numerical benchmark, we consider the following Dirichlet problem for the viscous Burgers equation, $A(x)=x^{2} / 2$ :

$$
\left\{\begin{array}{l}
\frac{\partial}{\partial t} v(t, x)=\frac{\partial^{2} v}{\partial x^{2}}(t, x)-v(t, x) \frac{\partial v}{\partial x}(t, x), t>0, x \in[0,2 \pi] \\
v(0, x)=\frac{2 \sin (x)}{\cos (x)+\mathrm{e}}, x \in[0,2 \pi] \text { and } \forall t \geq 0, v(t, 0)=0, v(t, 2 \pi)=0 .
\end{array}\right.
$$

The exact solution is $V(t, x)=2 \sin (x) /\left(\cos (x)+\mathrm{e}^{(1+\mathrm{t})}\right)$. Adapt the so far considered spatial domain [0,1] to $[0,2 \pi]$ is straightforward. A more significant modification concern the fact that the distribution derivative of $v(0, x)$ given by $U_{0}(x)=(2+2 \mathrm{e} \cos (x)) /(\cos (x)+\mathrm{e})^{2}$ is not a probability measure but a bounded signed measure. To take into account this modification, we use weighted particles $\left(Y_{t_{l}}^{i}, w^{i}\right)_{1 \leq i \leq N}$ (see section 2.3 and [19] which deals with a spatial domain equal to $\mathbb{R})$. The $N$ initial locations $y_{0}^{i}=\inf \{y ;(H *$ $\left.\left.\left.\left|U_{0}\right| /\left\|U_{0}\right\|_{\mathrm{L}^{1}([0,2 \pi])}\right)(y)=\frac{i}{N}\right\}\right)$ are chosen in order to approximate the cumulative distribution function of the probability measure $\left|U_{0}\right|(x) d x /\left\|U_{0}\right\|_{\mathrm{L}^{1}([0,2 \pi])}$ and the corresponding weights are $w_{i}=\left\|U_{0}\right\|_{\mathrm{L}^{1}([0,2 \pi])} \operatorname{sign}\left(U_{0}\left(y_{0}^{i}\right)\right)$. The approximate solution is given by the weighted cumulative distribution function of the particle system $\bar{V}\left(t_{l}, x\right)=\frac{1}{N} \sum_{i=1}^{N} w^{i} H\left(x-Y_{t_{l}}^{i}\right)$ where the successive positions are defined inductively by (54) but with $\wedge 1$ (respectively -1 ) replaced by $\wedge 2 \pi$ (resp. $-2 \pi$ ) in the second (resp. last) line. The parameters of Lépingle scheme are $\alpha_{0}=0.25$ and $\alpha_{1}=2 \pi-0.25$. The numerical solution at time $t=1$ is plotted on Figure 5 . The dependence of the error on the number of particles is standard and corresponds to the usual central limit theorem rate (see [9] [19] for numerical results when the spatial domain is $\mathbb{R}$ ). According to Theorem 5.7, $\mathbb{E}\|V(1, .)-\bar{V}(1, .)\|_{\mathrm{L}^{1}([0,2 \pi])} \leq 2 \pi \sup _{x \in[0,2 \pi]} \mathbb{E}|V(1, x)-\bar{V}(1, x)| \leq C\left(\Delta t+N^{-1 / 2}\right)$. Since it is not possible to compute the last quantity, we compute the first one by averaging $\|V(1, .)-\bar{V}(1, .)\|_{\mathrm{L}^{1}([0,2 \pi])}$ over 20 runs of 


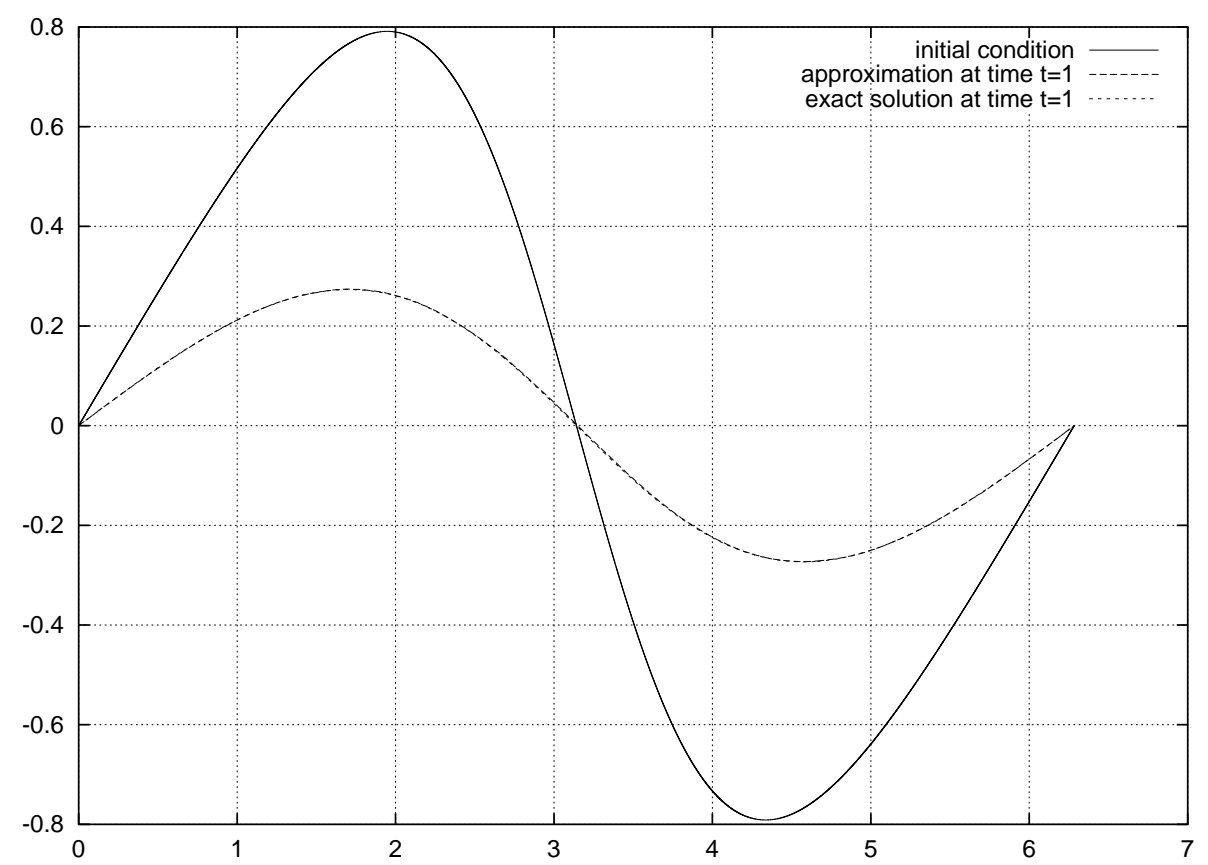

Figure 5. Exact and numerical solutions of (56) obtained at time $t=1$, for $10^{4}$ particles and $\Delta t=10^{-2}$ with the Lépingle scheme.

\begin{tabular}{|c||c|c||c|c|}
\hline$\Delta t$ & $\begin{array}{c}\text { Lépingle } \\
\text { scheme }\end{array}$ & $\begin{array}{c}\text { Confidence } \\
\text { interval at } 95 \%\end{array}$ & $\begin{array}{c}\text { Projection } \\
\text { scheme }\end{array}$ & $\begin{array}{c}\text { Confidence } \\
\text { interval at 95\% }\end{array}$ \\
\hline $2^{-1}$ & 0.0940 & {$[0.0933,0.0946]$} & 0.2510 & {$[0.2501,0.2519]$} \\
\hline $2^{-2}$ & 0.0585 & {$[0.0579,0.0591]$} & 0.2320 & {$[0.2309,0.2329]$} \\
\hline $2^{-3}$ & 0.0329 & {$[0.0322,0.0336]$} & 0.1964 & {$[0.1953,0.1975]$} \\
\hline $2^{-4}$ & 0.0173 & {$[0.0166,0.0180$} & 0.1568 & {$[0.1557,0.1578]$} \\
\hline $2^{-5}$ & 0.0083 & {$[0.0076,0.0090]$} & 0.1241 & {$[0.1227,0.1254]$} \\
\hline $2^{-6}$ & 0.0053 & {$[0.0045,0.0060]$} & 0.0982 & {$[0.0969,0.0995]$} \\
\hline $2^{-7}$ & 0.0049 & {$[0.0043,0.0055]$} & 0.0779 & {$[0.0765,0.0793]$} \\
\hline $2^{-8}$ & 0.0050 & {$[0.0042,0.0058]$} & 0.0635 & {$[0.0627,0.0643]$} \\
\hline
\end{tabular}

TABLE 4. Expectation of $\mathrm{L}^{1}$ norm of error at $t=1$ for $N=10^{6}$ particles $\left(\|V(\cdot, 1)\|_{\mathrm{L}^{1}([0,2 \pi])}=1.09\right)$

the particle method and give the dependence of the result on $\Delta t$ in Table 4 and Figure 6 . The test case (56) produce a significant rate of effective reflections: there are about $10 \%$ of the particles in $\left[0, \alpha_{0}\right] \cup\left[\alpha_{1}, 2 \pi\right]$ at each time-step. For these particles, we have to compute the correction term $C^{i}$ in (54). When we discretize the particle system according to the projected Euler scheme, which treats the reflection simply by projection onto $[0,1]$, we clearly observe a sub-linear convergence in $\Delta t$ (see Table 4 and Figure 6 ). The projected Euler scheme does not use the correction term $C$ whatever the position of the particle and its weak convergence rate is in 
$\mathcal{O}\left(\Delta t^{1 / 2}\right)$, (see [14]). The quasi-linear decreasing of the error for the Lépingle scheme confirms the theoretical analysis.

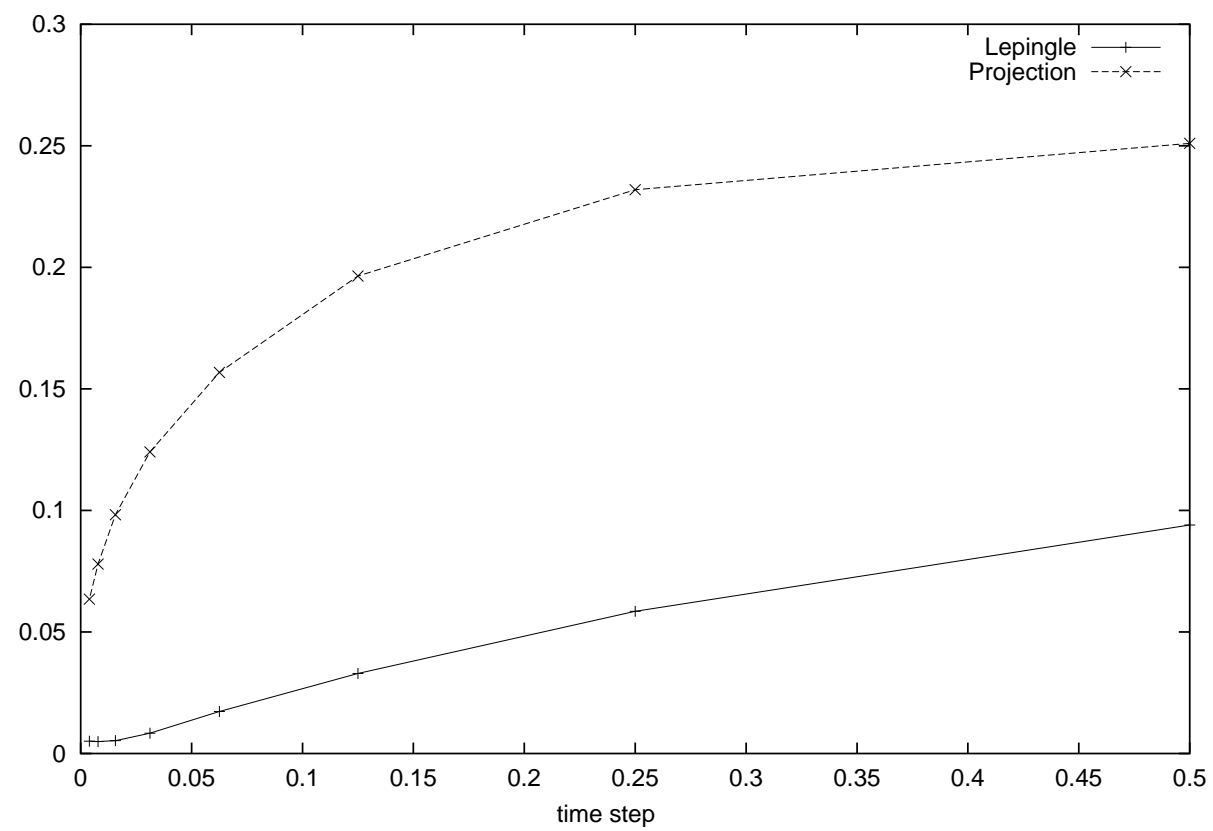

Figure $6 . \quad \mathbb{E}\|V(\cdot, 1)-\bar{V}(\cdot, 1)\|_{\mathrm{L}^{1}(\mathbb{R})}$ in terms of $\Delta t\left(N=10^{6}\right)$.

\section{REFERENCES}

[1] F. ANTONELLI and A. KOHATSU-HIGA. Rate of convergence of the particle method to the solution of the mc kean-vlasov's equation. Annals of Applied Probability, 12:423-476, 2002.

[2] J. T. BEALE and A. MAJDA. Vortex methods I: Convergence in three dimensions. Mathematics of Computation, 39:1-27, 1982.

[3] J. T. BEALE and A. MAJDA. Vortex methods II: Higher order accuracy in two and three dimensions. Mathematics of Computation, 39:29-52, 1982 .

[4] S. BENACHOUR, B. ROYNETTE, and P. VALLOIS. Branching process associated with 2d-Navier Stokes equation. Rev. Mat. Iberoamericana, 17(2):331-373, 2001.

[5] M. BOSSY. Optimal rate of convergence of a stochastic particle method to solutions of 1D viscous scalar conservation laws. Math. Comp., 73(246):777-812, 2004.

[6] M. BOSSY, L. FEZOUI, and S. PIPERNO. Comparison of a stochastic particle method and a finite volume deterministic method applied to burgers equation. Monte Carlo Methods and Appl., 3(2):113-140, 1997.

[7] M. BOSSY, E. GOBET, and D. TALAY. A symmetrized Euler scheme for an efficient approximation of reflected diffusions. J. Appl. Probab., 41(3):877-889, 2004.

[8] M. BOSSY and B. JOURDAIN. Rate of convergence of a particle method for the solution of a 1D viscous scalar conservation law in a bounded interval. Ann. Probab., 30(4):1797-1832, 2002.

[9] M. BOSSY and D. TALAY. A stochastic particle method for the McKean-Vlasov and the Burgers equation. Math. Comp., 66(217):157-192, 1997. 
[10] Y. BRENIER and GRENIER E. Sticky particles and scalar conservation laws. SIAM J. Numer. Anal., 35(6):2317-2328, 1998.

[11] P. CALDERONI and M. PULVIRENTI. Propagation of chaos for Burgers' equation. Ann. Inst. Henri Poincaré, 39(1):85-97, 1983.

[12] A.L. CHORIN. Numerical study of slightly viscous flow. J. Fluid Mech., 57(4):785-796, 1973.

[13] A.L. CHORIN. Vortex sheet approximation of boundary layers. J. Comp. Phys., 27:428-442, 1978.

[14] C. COSTANTINI, B. PACCHIAROTTI, and F. SARTORETTO. Numerical approximation for functionals of reflecting diffusion processes. SIAM J. Appl. Math., 58(1):73-102, 1998.

[15] G.H. COTTET. Boundary conditions and deterministic vortex methods for the Navier-Stokes equations. In Mathematical aspects of vortex dynamics (Leesburg, VA, 1988), pages 128-143. SIAM, Philadelphia, PA, 1989.

[16] J. FONTBONA. A probabilistic interpretation and stochastic particle approximations of the three dimensional navier-stokes equation. Probab. Theory Related Fields, To appear.

[17] J. GÄRTNER. On the Mckean-Vlasov limit for interacting diffusions. Math. Nachr., 137:197-248, 1988.

[18] J. GOODMAN. Convergence of the random vortex method. Comm. Pure Appl. Math., 40:189-220, 1987.

[19] B. JOURDAIN. Diffusion processes associated with nonlinear evolution equations for signed measures. Methodol. Comput. Appl. Probab., 2(1):69-91, 2000.

[20] B. JOURDAIN. Probabilistic characteristics method for a one-dimensional inviscid scalar conservation law. Ann. Appl. Probab., 12(1):334-360, 2002.

[21] B. JOURDAIN and S. MÉLÉARD. Probabilistic interpretation and particle method for vortex equations with Neumann's boundary condition. Proc. Edinb. Math. Soc. (2), 47(3):597-624, 2004.

[22] I. KARATZAS and S.E. SHREVE. Brownian Motion and Stochastic Calculus. Springer-Verlag, New York, 1988.

[23] A. KOHATSU-HIGA and S. OGAWA. Weak rate of convergence for an euler scheme of nonlinear sde's. Monte Carlo Methods and its Applications, 3:327-345, 1997.

[24] O.A. LADYZENSKAJA, V.A. SOLONNIKOV, and N.N. URAL'CEVA. Linear and Quasi-linear Equations of Parabolic Type, volume 23 of Transl. Math. Monographs. American Mathematical Society, 1988.

[25] C. LÉONARD. Une loi des grands nombres pour des systèmes de diffusions avec interaction et à coefficients non bornés. Ann. Inst. Henri Poincaré, 22(2):237-262, 1986.

[26] D. LEPINGLE. Euler scheme for reflected stochastic differential equations. Mathematics and Computers in Simulation, 38, 1995.

[27] D.G. LONG. Convergence of the random vortex method in two dimensions. J. Amer. Math. Soc., 1(4), 1988.

[28] C. MARCHIORO and M. PULVIRENTI. Hydrodynamics in two dimensions and vortex theory. Comm. Math. Phys., 84:483503, 1982.

[29] H.P. McKEAN. Propagation of chaos for a class of non-linear parabolic equation. Stochastic Differential Equations (Lecture Series in Differential Equations), 7:41-57, 1967.

[30] S. MÉLÉARD. Asymptotic behaviour of some interacting particle systems; McKean-Vlasov and Boltzmann models. In D. Talay and L. Tubaro, editors, Probabilistic Models for Nonlinear PDE's and Numerical Applications, Lecture Notes in Math., Berlin, Heidelberg, New York, 1996. Springer Verlag.

[31] S. MÉLÉARD. Monte-Carlo approximations for 2d Navier-Stokes equations with measure initial data. Probab. Theory Related Fields, 121(3):367-388, 2001.

[32] S. MÉLÉARD and S. ROELLY-COPPOLETTA. A propagation of chaos result for a system of particles with moderate interaction. Stochastic Process. Appl., 26:317-332, 1987.

[33] J.P. MINIER and E. PEIRANO. The pdf approach to turbulent polydispersed two-phase flows. Phys. Rep., 352(1-3):1-214, 2001.

[34] K. OELSCHLÄGER. A law of large number for moderately interacting diffusion processes. Z. Wahrsch. Verw. Geb., 69:279$322,1985$.

[35] H. OSADA. Propagation of chaos for the two dimensional Navier-Stokes equation. In K. Itô and N. Ikeda, editors, Probabilistic Methods in Mathematical Physics, pages 303-334. Academic Press, 1987. Proceedings of the Taniguchi International Symposium, Katata,1985.

[36] S.B. POPE. Application of the velocity-dissipation probability density function model to inhomogeneous turbulent flows. Phys. Fluids A, 3(8), 1991.

[37] S.B. POPE. Lagrangian PDF methods for turbulent flows. In Annual review of fluid mechanics, Vol. 26, pages 23-63. Annual Reviews, Palo Alto, CA, 1994.

[38] S.B. POPE. Lagrangian pdf methods for turbulent flows. Annu. Rev. Fluid Mech., 26:23-63, 1994.

[39] E.G. PUCKETT. Convergence of a random particle method to solutions of the Kolmogorov equation. Math. Comp., 52(186):615-645, 1989

[40] P.A. RAVIART. An analysis of particle methods. In F. Brezzi, editor, Numerical Methods in Fluid Dynamics, volume 1127 of Lecture Notes in Math., pages 243-324, Berlin, Heidelberg, New York, 1985. Springer-Verlag.

[41] A.S. SZNITMAN. A propagation of chaos result for Burgers' equation. Probability Theory, 71:581-613, 1986.

[42] A.S. SZNITMAN. Topics in propagation of chaos. In P.L. Hennequin, editor, Ecole d'Eté de Probabilités de Saint-Flour XIX - 1989, volume 1464 of Lecture Notes in Math., pages 165-251, Berlin, Heidelberg, New York, 1991. Springer-Verlag. 
[43] D. TALAY. Probabilistic numerical methods for partial differential equations: elements of analysis. In D. Talay and L. Tubaro, editors, Probabilistic Models for Nonlinear Partial Differential Equations, volume 1627 of Lecture Notes in Mathematics, pages 148-196. Springer-Verlag, 1996.

[44] D. TALAY and L. TUBARO. Expansion of the global error for numerical schemes solving stochastic differential equations. Stoch. Anal. Appl., 8(4):94-120, 1990.

[45] H. TANAKA. Limit theorems for certain diffusion processes with interaction. In Stochastic analysis (Katata/Kyoto, 1982), volume 32 of North-Holland Math. Library, pages 469-488. North-Holland, Amsterdam, 1984. 